\title{
Nanosatellite-5G Integration in the Millimeter Wave Domain: A Full Top-Down Approach
}

\author{
F. Babich ${ }^{\dagger}$, Senior Member, IEEE, M. Comisso ${ }^{\dagger}$, Member, IEEE, A. Cuttin ${ }^{\dagger}$, Member, IEEE, \\ M. Marchese ${ }^{\S}$, Senior Member, IEEE, and F. Patrone ${ }^{\S}$, Member, IEEE \\ ${ }^{\dagger}$ Dept. of Engineering and Architecture (DIA), Univ. of Trieste (Italy)

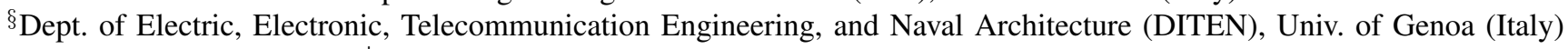 \\ E-mail: ${ }^{\dagger}\{$ babiche, mcomisso@, alessandro. cuttin@phd. $\}$ units.it \\ $\S\{$ mario.marchesed, f.patronededu. $\}$ unige.it
}

\begin{abstract}
This paper presents a novel network architecture for an integrated nanosatellite (nSAT)-5G system operating in the millimeter-wave (mmWave) domain. The architecture is realized adopting a delay/disruption tolerant networking (DTN) approach allowing end users to adopt standard devices. A buffer aware contact graph routing algorithm is designed to account for the buffer occupancy of the nSATs and for the connection planning derived from their visibility periods. At the terrestrial uplink, a coded random access is employed to realize a high-capacity interface for the typically irregular traffic of $5 \mathrm{G}$ users, while, at the space uplink, the DTN architecture is combined with the contention resolution diversity slotted Aloha protocol to match the recent update of the DVB-RCS2 standard. To achieve a reliable testing of the introduced functionalities, an accurate analysis of the statistic of the signal to interference-plus-noise ratio and of the capture probability at each mmWave link is developed by including interference, shadowing, fading, and noise. The application of the designed architecture to data transfer services in conjunction with possible delay reduction strategies, and an extension to inter-satellite communication, are finally presented by estimating the resulting loss/delay performance through a discrete-time discrete-event platform based on the integration of Matlab with Network Simulator 3.
\end{abstract}

Index Terms-Nanosatellite-5G integration; delay/disruption tolerant network; contact graph routing; uncoordinated access; capture probability; millimeter-wave.

\section{INTRODUCTION}

Initially conceived for scientific and educational purposes, nanosatellites (nSATs) rapidly gained the interest of commercial and government entities for the cheapness of the components and the availability of launches at reduced cost. At this early stage, the basic applications enabled by small satellite designers were mainly related to environmental monitoring and educational missions, thus the bands assigned to the amateur radio service were considered sufficient. The recent entrance of military and commercial subjects has instead changed this perspective, determining the need of larger transfer rates [1]. To deal with this issue, spacecraft systems' developers and scientists have recently identified the exploitation of the millimeter wave (mmWave) bands, between 30 and $300 \mathrm{GHz}$, as a

\footnotetext{
${ }^{1}$ This work is partly supported by the European Space Agency (ESA) within the project SatNEx IV CoO 1 Part 2 entitled "Multi-homed network architectures for flying ad-hoc networks and nanosatellite swarms", and by the Italian Ministry of University and Research (MIUR) within the project FRA 2018 (University of Trieste, Italy), entitled "UBER-5G: Cubesat 5G networks - Access layer analysis and antenna system development".
}

key point to satisfy the huge capacity demand of forthcoming nSAT networks [1], [2]. Previously used mainly for large satellites, mmWaves are in fact now under consideration also for the low-Earth orbit (LEO) ones. The motivation of this choice lies in the considerable advantages offered by the extremely high frequencies (EHF) in terms of higher reuse factor and larger bandwidth with respect to the conventional $\mathrm{S}$ and $\mathrm{X}$ bands. Moreover, the applicability of the mmWave technology to the small satellite context is nowadays made possible by the availability of compact high-gain K-band antennas [3], [4], and of EHF radio transceivers satisfying power/size constraints and providing rates in the order of hundreds of megabits [5], [6].

Beside this evolution in spacecraft communications, another technological outbreak is expected to arrive from the realization of the next-generation $5 \mathrm{G}$ terrestrial network, in which mmWaves represent, together with densification and massive multiple input multiple output [7], the "big three" requirements to overcome the capacity limitations of current microwave cellular systems [8]. Among these requirements, the $5 \mathrm{G}$ densification strategy relies on the deployment of a huge number of base stations (BSs), whose installation can be highly remunerative in the urban areas, but may result not profitable in the remote ones. In this second situation, a fundamental support to the communications between far $5 \mathrm{G}$ BSs may be provided by nSAT constellations, thus suggesting the development of an integrated nSAT-5G network. The interest for a hybrid nSAT-5G network finds its main reason in the intrinsic ubiquity and broadcasting capabilities of satellites, which may play multiple roles in $5 \mathrm{G}$, such as the support of edge computing services, the backhauling for rural areas, and the geographical distribution of application software. The resulting outcomes are expected to have a significant impact on many emerging paradigmatic use cases, such as smart farms, smart cities, and smart industries, for a variety of applications, including remote monitoring of agricultural fields, traffic offloading, and sensors/actuators control in dangerous environments [9].

\section{A. Related work}

The problem of integrating nSAT and 5G technologies for mobile broadband coverage has already attracted the attention 
of the satellite research community [10]-[13]. In particular, the application of the cognitive radio paradigm to an nSAT-5G network has been addressed in [10], where a software-defined radio (SDR) platform has been developed to investigate the access and frequency allocation issues. A dynamic hybrid terrestrial-satellite backhaul system has been proposed in [11], by considering the functionalities that must be introduced at the physical (PHY), medium access control (MAC), and network (NET) layers to enable the reconfiguration of the terrestrial topology. An end-to-end satellite-terrestrial network has been presented in [12], by providing a detailed theoretical analysis of the coverage probability and of the spectral and energy efficiencies for different node densities. An SDR architecture for next-generation satellite networks, further including the software-defined networking (SDN) and network function virtualization (NFV) paradigms, has been described in [13], together with a possible roadmap for its deployment and integration with the $5 \mathrm{G}$ infrastructure. The co-channel interference at 28 and $70 \mathrm{GHz}$ between $5 \mathrm{G}$ systems and mmWave fixed satellite services (FSSs) has been studied in [14], showing that protection mechanisms may be introduced in the $5 \mathrm{G}$ devices to mitigate the interference towards the FSS without significantly affecting the $5 \mathrm{G}$ network performance.

Outstanding results have been recently achieved also on random multiple access for cellular and satellite networks, where the need of managing inhomogeneous traffic conditions and the impossibility of using carrier sensing mechanisms have determined the adoption of the slotted-Aloha (SA) scheme. In this context, the introduction of interference cancellation (IC) has enabled the design of the contention resolution diversity SA (CRDSA) protocol [15], a high-capacity iterative repetition-based algorithm included in the last DVB-RCS2 standard [16], and of the coded SA (CSA) scheme [17], an energy-efficient iterative solution presently under consideration for the uplink random access of forthcoming $5 \mathrm{G}$ terrestrial cellular systems [18].

From the point of view of an integrated nSAT-5G system, it may be really fruitful to add these novel algorithms into a delay/disruption tolerant networking (DTN) architecture [19], [20], which has been already successfully applied to challenging LEO satellite scenarios for data exchange purposes [21]. The DTN concept, which operates through a store and forward mechanism, is based on the introduction of an overlay layer directly above the transport or the lower-layer protocols. Nodes with such an overlay layer are able to handle delays and disruptions by storing the application data inside their buffer until the next hop becomes available [22]. Simultaneous end-to-end connectivity is hence not required, thus making the DTN solution specifically suited for integrated nSAT$5 \mathrm{G}$ communication networks. In the small satellite context, a DTN-based architecture has been proposed in [23], as a possible solution for integrating nSAT networks and terrestrial infrastructures to extend Internet connectivity to rural and remote areas. Equipping satellites with inter-satellite links (ISLs) can further contribute to improve the network performance, without making the DTN paradigm pointless. In this case, an exhaustive overview of the suitable architectures and protocols is presented in [24].

\section{B. Contribution and organization}

In light of the above considerations, this paper proposes a top-down network architecture for an integrated nSAT-5G system operating in the mmWave domain. Three are the main original contributions of this study. The first contribution concerns the derivation of a novel PHY layer theoretical analysis that provides the distribution of the signal to interference-plusnoise ratio (SINR) at each mmWave link in a propagation environment characterized by shadowing, fading, interference, and noise. The second contribution involves the revision of the performance of the CSA and CRDSA random access schemes in the presence of capture by adopting advanced reception criteria adherent to the estimated SINR distributions. The third contribution consists in the design of a novel buffer aware contact graph routing (BA-CGR) algorithm for the DTN layer and the definition of an extension block of the bundle protocol (BP). The aim of this novel algorithm is to account for the buffer occupancy and the visibility periods of the nSATs, so as to forward and exploit information about the storing capabilities of the intermediate nodes and the amount of data that can be exchanged during each contact. The conceived network architecture is implemented on a discrete-time discrete-event simulator based on the combination of Matlab with Network Simulator 3 (NS3). This integrated Matlab-NS3 platform is used to investigate the applicability of the nSAT-5G system to data transport services with different loss/delay requirements, including, as a possible final extension, the presence of ISLs.

The paper is organized as follows. The scenario is introduced in Section II. The PHY layer is analyzed in Section III. The MAC layer procedures are addressed in Section IV. The designed BA-CGR algorithm is presented in Section V. The numerical results are discussed in Section VI. The conclusions are summarized in Section VII.

\section{SCENARIO}

The reference end-to-end communication scenario is shown in Fig. 1, which also reports the meaning of the mathematical symbols that will be used for the PHY layer analysis. According to this figure, consider a $5 \mathrm{G}$ cell shared by $N$ mobile stations (MSs) that operate as data sources, where $\mathrm{M}_{n}$ $(n=1, \ldots, N)$ denotes the generic source. This cell is covered by a target $\mathrm{BS} \mathrm{B}_{L}$ belonging to a set of $L$ BSs that can also establish a connection with a generic $\operatorname{nSAT~}_{q}(q=1, \ldots, Q)$, which is part of a constellation of $Q$ nSATs. Within this constellation, only one nSAT at a time is visible to the $L$ BSs. To establish the connection, the generic $\mathrm{BS}_{l}(l=1, \ldots, L)$ is equipped with two mmWave radio interfaces: a terrestrial $5 \mathrm{G}$ one and a satellite one, thus it can operate as an nSAT-5G gateway. When a target $\mathrm{MS} \mathrm{M}_{N}$ has to communicate with a destination MS $\mathrm{M}^{\prime}$, lying in a cell managed by a $5 \mathrm{G}$ BS $\mathrm{B}^{\prime}$ covered by the orbit of $\mathrm{S}_{q}, \mathrm{~S}_{q}$ acts as relay. In particular, $\mathrm{S}_{q}$ uploads the data from $\mathrm{B}_{L}$ during the $\mathrm{B}_{L}-\mathrm{S}_{q}$ visibility period and downloads them to $\mathrm{B}^{\prime}$ during the $\mathrm{S}_{q}-\mathrm{B}^{\prime}$ visibility period. Hence, the $\mathrm{M}_{N}-\mathrm{M}^{\prime}$ end-to-end connection involves four links: the terrestrial uplink (TU) $\mathrm{M}_{N}-\mathrm{B}_{L}$, the space uplink (SU) $\mathrm{B}_{L}-\mathrm{S}_{q}$, the space downlink (SD) $\mathrm{S}_{q}-\mathrm{B}^{\prime}$, and the terrestrial downlink (TD) $\mathrm{B}^{\prime}-\mathrm{M}^{\prime}$. 


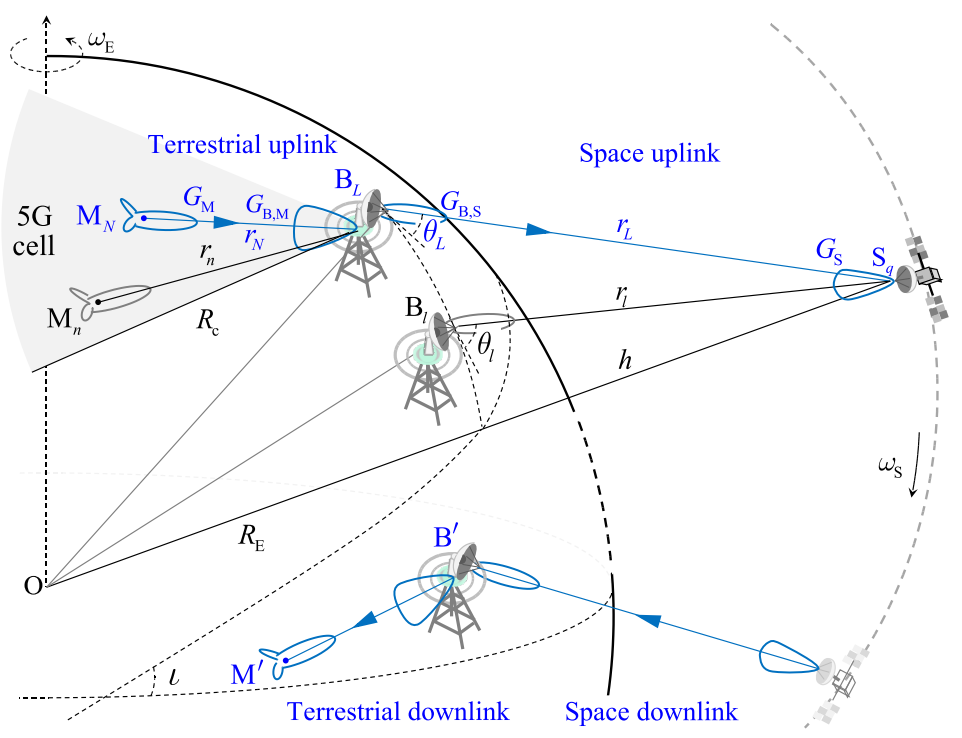

\begin{tabular}{|c|}
\hline $\mathrm{M}_{n}$ : generic MS (5G source) \\
\hline $\mathrm{B}_{l}$ : generic BS (nSAT-5G gateway) \\
\hline $\mathrm{S}_{q}:$ generic nSAT \\
\hline $\mathrm{B}^{\prime}$ : destination BS \\
\hline $\mathrm{M}^{\prime}$ : destination MS \\
\hline $\mathrm{M}_{N}-\mathrm{B}_{L}-\mathrm{S}_{q}-\mathrm{B}^{\prime}-\mathrm{M}^{\prime}$ : target connection \\
\hline$R_{\mathrm{c}}:$ cell radius \\
\hline$r_{n}: \mathrm{M}_{n}-\mathrm{B}_{L}$ distance \\
\hline$G_{\mathrm{M}}: \mathrm{MS}$ antenna gain \\
\hline $\begin{aligned} G_{\mathrm{B}, \mathrm{M}}: & \mathrm{BS} \text { antenna gain } \\
& \text { (terrestrial interface) }\end{aligned}$ \\
\hline $\begin{array}{r}G_{\mathrm{B}, \mathrm{S}}: \\
\begin{array}{c}\text { BS antenna gain } \\
\text { (space interface) }\end{array}\end{array}$ \\
\hline$r_{l}: \mathrm{B}_{l}-\mathrm{S}_{q}$ distance \\
\hline$\theta_{l}: \mathrm{B}_{l}-\mathrm{S}_{q}$ elevation angle \\
\hline$\iota:$ inclination angle \\
\hline$h:$ orbit altitude \\
\hline$R_{\mathrm{E}}:$ Earth radius \\
\hline$G_{\mathrm{S}}: \mathrm{nSAT}$ antenna gain \\
\hline$\omega_{\mathrm{S}}:$ nSAT angular velocity \\
\hline$\omega_{\mathrm{E}}$ : Earth rotation angular velocity \\
\hline
\end{tabular}

Fig. 1. End-to-end communication scenario.

Even if the depicted scenario represents a common application for an integrated terrestrial-satellite network, it is characterized by some specific features that require a careful design of the network architecture. In fact, since LEO satellites move on subsynchronous orbits, their footprints are not the same during the orbit time, thus, in most cases, $\mathrm{B}_{L}$ and $\mathrm{B}^{\prime}$ are not covered by the same satellite at the same time. Nevertheless, also in these cases the end-to-end connection remains mandatory for a lot of transport protocols used in classical networks. Besides, the technology that must be managed is necessarily heterogeneous, since $5 \mathrm{G}$ and satellite devices are involved, and the end-to-end connection is composed by a concatenation of time-disjoint transient links. The terrestrial links between MSs and BSs may be generally assumed active except for unpredictable and temporary faults. The satellite links between BSs and nSATs may be unavailable for extended periods of time and subject to time-varying propagation delays. Consequently, there are storage constraints both at BSs and nSATs, since the packets have to be forwarded through the satellite link only during the visibility periods. Moreover, the uplinks have to implement random access mechanisms to cope with the unpredictability of the sources' activity, while the downlinks may be reasonably assumed to rely on a reserved access. Furthermore, the strong attenuations that characterize the mmWave links need to be considered, also because their effects may be further exacerbated by the disruptions due to the satellite movement and by the delays due to the long distances between nSATs and BSs.

\section{A. Architecture}

To deal with the above discussed issues, this paper proposes a novel network architecture combining the DTN paradigm with coded random access (Fig. 2). In this architecture, the MSs are regular Internet hosts implementing a user datagram protocol / transfer control protocol - Internet protocol (UDP/TCP-IP) stack; the nSATs are DTN nodes implementing the DTN architecture through a bundle layer (BL); while the BSs provide a UDP/TCP-IP interface towards the MSs and a DTN interface towards the nSATs [25]. Thus, the BSs act as DTN gateways and implement a relay function that performs the bundle encapsulation/de-encapsulation procedures. In particular, the term bundle here refers to a message composed of a payload containing the application data and of a header containing the protocol control information necessary for the end-to-end delivery. The BL is hence directly interfaced with the MAC layer used for the satellite links both at BSs and nSATs. In short, the DTN architecture is composed of heterogeneous areas where intermediate nodes (BSs and nSATs) are DTN-based. MSs are UDP/TCP-IP based devices not equipped with the BP, avoiding the need of ad-hoc devices or the implementation of software modifications. TCP support can be guaranteed through performance enhancing proxies (PEPs) based on TCP-splitting. They can split TCP connections in more parts, in order to isolate the satellite link characteristics from the rest of the network [21]. In this scenario, TCP connections would be split in two portions: the first one between source MSs and source BSs, and the second one between destination BSs and destination MSs.

The BL implements the proposed BA-CGR algorithm, which enables the correct packet forwarding to the destination through the complete knowledge of the future contacts [26], additionally accounting for the nSAT buffer occupancy. This latter element is in fact more critical for nSATs than for BSs, since the formers have to satisfy more stringent size and weight constraints. The purpose of BA-CGR is to avoid nSAT buffer overflows and routing path calculations towards future contacts without enough remaining space. At the lower layers, the multiple access algorithms are selected in agreement with current 5G and nSAT proposals. Accordingly, the MAC layer for the downlinks relies on a demand assigned multiple access (DAMA), while the one for the uplinks relies on a contentionbased approach to manage the typical irregular traffic of the 


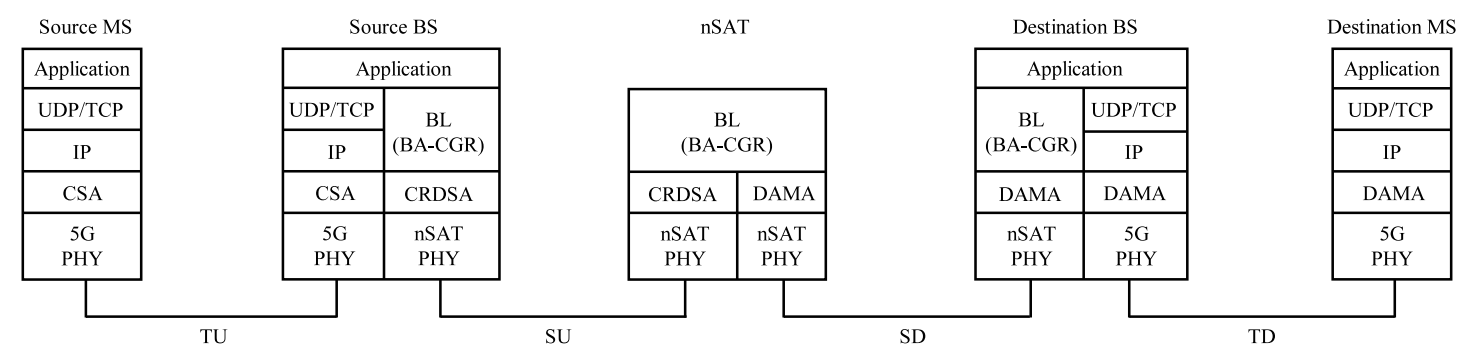

Fig. 2. Network architecture.

terrestrial users. More precisely, the CSA scheme is selected for the random access at the TU to provide a high-capacity interface [17], [18], while the CRDSA protocol is chosen to manage the random access at the $\mathrm{SU}$ to match the recent upgrade of the DVB-RCS2 standard [15], [16]. Both schemes combine IC with proper diversity (CRDSA) and coding (CSA) strategies to increase the MAC layer throughput.

In summary, the data packets generated by the application layer of the source MS $\mathrm{M}_{N}$ are sent, through a UDP/TCP-IP interface, to the source BS $\mathrm{B}_{L}$ by the CSA scheme. Then, $\mathrm{B}_{L}$ encapsulates the packets in data bundles storing them in its buffer, and, through the CRDSA scheme, uploads the bundles on the nSAT selected by the BA-CGR algorithm. The designed $\mathrm{BL}$ also ensures the transmission of the source/destination IP addresses so as to guarantee the identification of the source/destination MSs. The chosen nSAT stores the received bundles in its buffer until it enters in contact with $\mathrm{B}^{\prime}$, which de-encapsulates them, gets the IP addresses, and sends the corresponding packets to $\mathrm{M}^{\prime}$. In this way, we get a transparent bidirectional communication between each pair of MSs. It is important to remark the relay action of the BL, which allows not only the information storage at intermediate nodes, but also the implementation of dedicated protocol stacks for single network portions.

\section{B. Relationships with $5 G$ functionalities}

Several elements of the considered scenario can be mapped into the upcoming $5 \mathrm{G}$ architecture. For example, a BS might be viewed by the MSs as a gNodeB (gNB) with an additional satellite interface and DTN gateway capabilities. In this sense, the 5G-PHY and CSA MAC layers may be considered part of a gNB distributed unit (DU), according to most of the current options proposed by 3GPP [27]. An exhaustive analysis of all the possibilities is anyway a rather difficult task, because several options are still under evaluation for the splitting of the MAC/PHY functionalities between the central unit (CU) and the DU. Among them, we may however signal the adoption of a radio link control (RLC)-MAC split, in which, radio resource control (RRC), packet data convergence protocol (PDCP), and RLC are in the CU, while, as previously outlined, CSA-MAC and PHY are in the DU. Another possibility is an intra MAC split in which radio frequency (RF), PHY and the lower part of the MAC layer (Low-MAC) are in the DU, while RLC, PDCP, and the higher part of the MAC layer (High-MAC) are in the CU. By splitting the MAC layer into two entities, its services and functions may be located in the $\mathrm{CU}$, the $\mathrm{DU}$, or in both. The High-MAC sublayer may be in charge of the control of multiple Low-MAC sublayers, taking centralized scheduling decision and inter-cell interference coordination. In Low-MAC sublayer the time-critical functions, such as CSA, may be performed.

Many functionalities could be also implemented exploiting the NFV and SDN paradigms. Referring to the ETSI NFV management and orchestration (MANO) [28], current satellite networking elements (both BSs and nSATs) can be seen as physical network functions (PNFs). BSs' functionalities, such as packet encapsulation/de-encapsulation and routing path computation, could be virtualized except for the outdoor unit (ODU), which remains a PNF. After virtualization, these functionalities need to be integrated and orchestrated as slice components by an NFV-orchestrator (NFVO), which has to be located in a central node of the network, e.g., the satellite network and operational control center (NOCC). In this way, they could be remotely executed outside the BSs and opportunely tuned depending on the vertical applications' requirements coming from the operational support system / business support system (OSS/BSS). Both physical and virtual resources can be employed by the wide area network (WAN) infrastructure manager (WIM) whenever needed to support applications whose key performance indicators (KPIs) are compatible with their characteristics. The role of SDN here becomes instrumental to allow fast reconfiguration and interconnection of different allocated resources that can belong to different physical nodes. More details about SDN, NFV, and slicing concepts implementation in satellite networks, related open challenges and a possible roadmap, can be found in [29].

A further observation concerning the designed nSAT-5G architecture may be formulated by considering its matching with the typical 5G application categories [30]. In particular, massive machine-type communications (mMTC) can be properly sustained, since this $5 \mathrm{G}$ application is characterized by a large number of connected devices exchanging non-delaysensitive data. Besides, the wide-area coverage and the high traffic capacity requirements of enhanced mobile broadband (eMBB) can be also suitably supported, thanks to exploitation of the mmWave band. Instead, ultra-reliable low-latency communication (URLLC) does not properly comply with the conceived system, because of the low latency constraint, which is difficult to satisfy for the possible lack of endto-end paths between source and destination MSs, and the 
consequent long-time storage on-board nSATs. The latency, however, represents a common limit of satellite networks in general, which have hence an impact also when integrated space-terrestrial solutions are designed.

A detailed characterization of the developed architecture is presented in the next sections through a bottom-up approach involving the description of the PHY (Section III), MAC (Section IV), and NET (Section V) layers.

\section{PHY LAYER}

From a PHY layer point of view, each link of the $\mathrm{M}_{N^{-}}$ $\mathrm{M}^{\prime}$ end-to-end connection is characterized by a SINR statistic requiring a specific estimation. To this aim, some reasonable assumptions are introduced to limit the complexity of the analysis and avoid cumbersome notations.

Firstly, during a contact, an nSAT follows a circular orbit, thus its angular velocity in an Earth-centered Earth-fixed (ECEF) coordinate system is constant and given by [31]:

$$
\omega \cong \sqrt{\frac{\mu_{\mathrm{E}}}{\left(R_{\mathrm{E}}+h\right)^{3}}}-\omega_{\mathrm{E}} \cos \iota=\omega_{\mathrm{S}}-\omega_{\mathrm{E}} \cos \iota,
$$

where $\mu_{\mathrm{E}}$ is the geocentric gravitational constant, $R_{\mathrm{E}}$ is the Earth radius, $h$ is the orbit altitude, $\omega_{\mathrm{E}}$ is the angular velocity of the Earth rotation, $\iota \in\left[0^{\circ}, 90^{\circ}\right]$ is the inclination angle, and $\omega_{\mathrm{S}}$ is the angular velocity of the nSAT in an Earth-centered inertial (ECI) coordinate system. A BS $\mathrm{B}_{l}$ sees the nSAT from an elevation angle $\theta_{l}$ lying between a minimum value $\bar{\theta}_{1}$ and a maximum one $\bar{\theta}_{2}$, with $0^{\circ} \leq \bar{\theta}_{1} \leq \bar{\theta}_{2} \leq 90^{\circ}$. In these conditions, the visibility period is given by [31]:

$$
v=2 \zeta / \omega
$$

where $\left.\zeta=\arccos \left(\bar{\eta}_{1} / \bar{\eta}_{2}\right), \bar{\eta}_{i}=\cos \left[\gamma\left(\bar{\theta}_{i}\right)\right]\right\}$ for $i=1,2$, and:

$$
\gamma(x)=\arccos \left(\frac{R_{\mathrm{E}}}{R_{\mathrm{E}}+h} \cos x\right)-x .
$$

Secondly, the transmission powers employed at the terrestrial and space links are identified by $P_{\mathrm{tE}}$ and $P_{\mathrm{tS}}$, respectively, while all receivers are characterized by the same noise figure $\mathcal{F}$ and bandwidth $B_{\mathrm{W}}$. Therefore, denoting as $\chi_{\mathrm{B}}$ the Boltzmann constant, the noise powers at a terrestrial and satellite receiver may be evaluated, respectively, as [32]:

$$
\begin{aligned}
& \rho_{\mathrm{E}}=\chi_{\mathrm{B}} T_{\mathrm{E}} B_{\mathrm{W}} \mathcal{F}, \\
& \rho_{\mathrm{S}}=\chi_{\mathrm{B}} T_{\mathrm{S}} B_{\mathrm{W}} \mathcal{F},
\end{aligned}
$$

where $T_{\mathrm{E}}$ and $T_{\mathrm{S}}$ are the terrestrial and satellite antenna temperatures, respectively.

Thirdly, all channels are symmetric. To enable a compact analytical treatise, a Friis transmission model is adopted for the path-loss, thus considering a path-loss exponent equal to 2 on all links. This hypothesis is consistent with recent measurements in 5G line-of-sight (LOS) scenarios [33]. The space links are also subject to fast-fading and additional atmospheric attenuations [32], while the terrestrial links to mid-scale fading [33]. Each uplink is further affected by interference due to the contention process during the data upload, but not to the traffic on the other uplink, since non-overlapping frequency bands are assumed for the TU and SU.
According to these hypotheses, the following subsections provide estimations for the SINR distributions on the four links, with the purpose of getting a reliable physical basis for the application of the functionalities introduced at the upper MAC and DTN layers.

\section{A. Terrestrial links}

As discussed above, the $5 \mathrm{G}$ TU is subject to path-loss, shadowing, interference, and noise. The first two phenomena directly influence the power $p_{n}$ received by the target BS from the generic $\mathrm{MS} \mathrm{M}_{n}$. This quantity may be hence expressed as:

$$
p_{n}=\frac{P_{\mathrm{tE}} G_{\mathrm{M}} G_{\mathrm{B}, \mathrm{M}}}{\alpha r_{n}^{2}} \xi=\frac{\chi_{\mathrm{E}}}{r_{n}^{2}} \xi, \quad n=1, \ldots, N
$$

where $G_{\mathrm{M}}$ and $G_{\mathrm{B}, \mathrm{M}}$ are the maximum antenna power gains of a MS, and of a BS at its terrestrial radio interface, respectively; the distance $r_{n}$ is the realization of a random variable (r.v.) derived from a uniform distribution (u.d.) inside a disk of radius $R_{\mathrm{c}}$ (Fig. 1); $\alpha$ is the floating intercept of the pathloss model; $\xi$ is the realization of a log-normal r.v.; and $\chi_{\mathrm{E}}=P_{\mathrm{tE}} G_{\mathrm{M}} G_{\mathrm{B}, \mathrm{M}} / \alpha$. The modeling approach that leads to (5) directly derives from recent channel measurements [33], where path-loss attenuation and log-normal shadowing have been proved to be of main relevance in $5 \mathrm{G}$ communications [35]. The $\mathrm{BS}_{L}$ adopts a multi-beam antenna pattern to cover the entire $5 \mathrm{G}$ cell (Fig. 1 reports only one sector of the cell to provide a clearer figure), thus simultaneously receiving the power from all the $N$ contending MSs present in the cell. By consequence, the SINR at the TU $\mathrm{M}_{N}-\mathrm{B}_{L}$ is evaluated as:

$$
s=\frac{p_{N}}{\sum_{n=1}^{N-1} p_{n}+\rho_{\mathrm{E}}} .
$$

Observe that (6) is formulated according to the independent shadowing assumption [35], which states that the possible correlation among the different links has a minor impact on the SINR distribution [36]. Therefore, the different links (desired and interfering) may be reasonably considered independent, and hence the overall interference experienced by the $\mathrm{M}_{N}-\mathrm{B}_{L}$ communication may be estimated as the sum of $N-1$ uncorrelated powers $p_{1}, \ldots, p_{N-1}$. The noise $\rho_{\mathrm{E}}$ is also included in (6) because, differently from conventional microwave networks, which are usually interference-limited, mmWave networks may be interference-limited or noise-limited, depending on the specific environment and on the interferers' density [37]. A proper mathematical analysis of this scenario allows one to formulate the following proposition.

Proposition 1: The complementary cumulative distribution function (CCDF) of the SINR for the TU when $N$ contending MSs are present may be estimated as:

$$
\bar{F}_{S}^{\mathrm{TU}}(s ; N) \cong \frac{1}{2^{N}} \int_{s \rho_{\mathrm{E}}}^{+\infty} \frac{I_{1}\left(p_{N}\right)}{p_{N}}\left[\sum_{j=0}^{1} I_{j}\left(\frac{p_{N}-s \rho_{\mathrm{E}}}{s H_{N-1}}\right)\right]^{N-1} \mathrm{~d} p_{N},
$$

where $H_{\kappa}=\sum_{\kappa^{\prime}=1}^{\max (\kappa, 1)} 1 / \kappa^{\prime}$ coincides with the harmonic number of order $\kappa$ when $\kappa \in \mathbb{N}^{+}$, and:

$$
I_{j}(x)=\left(-\frac{\chi_{\mathrm{E}}}{R_{\mathrm{c}}^{2} x}\right)^{j} e^{j \frac{\sigma^{2}}{2}} \operatorname{erfc}\left[\frac{1}{\sqrt{2} \sigma} \log \left(\frac{\chi_{\mathrm{E}}}{R_{\mathrm{c}}^{2} x}\right)+\frac{j \sigma}{\sqrt{2}}\right],
$$




\begin{tabular}{|l|l|l|l|l|l|}
\hline$\mu_{\mathrm{E}} \cong 398600 \mathrm{~km}^{3} / \mathrm{s}^{2}$ & $c \cong 3.00 \cdot 10^{8} \mathrm{~m} / \mathrm{s}$ & $T_{\mathrm{E}}=270 \mathrm{~K}$ & $\alpha=61.4 \mathrm{~dB}$ & $\iota=50^{\circ}$ & $G_{\mathrm{S}}=30 \mathrm{~dB}$ \\
\hline$R_{\mathrm{E}} \cong 6371 \mathrm{~km}$ & $B_{\mathrm{W}}=450 \mathrm{MHz}$ & $R_{\mathrm{c}}=100 \mathrm{~m}$ & $\sigma=5.8 \mathrm{~dB}$ & $\bar{\theta}_{1}=10^{\circ}$ & $\nu=27.2 \mathrm{GHz}$ \\
\hline$\omega_{\mathrm{E}} \cong 7.29 \cdot 10^{-5} \mathrm{rad} / \mathrm{s}$ & $\mathcal{F}=10 \mathrm{~dB}$ & $G_{\mathrm{M}}=24 \mathrm{~dB}$ & $T_{\mathrm{S}}=100 \mathrm{~K}$ & $\bar{\theta}_{2}=90^{\circ}$ & $A=4 \mathrm{~dB}$ \\
\hline$\chi_{\mathrm{B}} \cong 1.38 \cdot 10^{-23} \mathrm{~J} / \mathrm{K}$ & $P_{\mathrm{tE}}=P_{\mathrm{tS}}=1 \mathrm{~W}$ & $G_{\mathrm{B}, \mathrm{M}}=24 \mathrm{~dB}$ & $h=400 \mathrm{~km}$ & $G_{\mathrm{B}, \mathrm{S}}=50 \mathrm{~dB}$ & $m=2$ \\
\hline
\end{tabular}

TABLE I

PHY LAYER PARAMETERS [33], [34].

in which $\sigma$ is the shadowing standard deviation and $\operatorname{erfc}(\cdot)$ is the complementary error function.

Proof: See Appendix A.

This proposition includes also the TD case. In fact, the channel symmetry, the antenna reciprocity, and the absence of interference due to the usage of the DAMA protocol for the downlink communications, imply that the CCDF of the SINR for the TD may be directly obtained from (7) as:

$$
\bar{F}_{S}^{\mathrm{TD}}(s)=\bar{F}_{S}^{\mathrm{TU}}(s ; 1) .
$$

\section{B. Space links}

The mmWave SU is subject to path-loss and atmospheric attenuations, fast-fading, interference, and noise. In this case, the distance $r_{l}$ between the nSAT and the $\mathrm{BS} \mathrm{B}_{l}$ (slant range), may be evaluated from geometrical considerations (Fig. 1), as:

$$
r_{l}=\sqrt{h^{2}+2 h R_{\mathrm{E}}+R_{\mathrm{E}}^{2} \sin ^{2} \theta_{l}}-R_{\mathrm{E}} \sin \theta_{l} .
$$

Given this relationship, the power $p_{l}$ received by the nSAT from $\mathrm{B}_{l}$ can be calculated as:

$$
p_{l}=\frac{P_{\mathrm{tS}} G_{\mathrm{B}, \mathrm{S}} G_{\mathrm{S}}}{A}\left(\frac{c}{4 \pi \nu r_{l}}\right)^{2} \psi=\frac{\chi_{\mathrm{S}}}{r_{l}^{2}} \psi, \quad l=1, \ldots, L
$$

where $G_{\mathrm{B}, \mathrm{S}}$ is the maximum antenna power gain of $\mathrm{B}_{l}$ at its space radio interface, $G_{\mathrm{S}}$ is the maximum antenna power gain of the nSAT, $A$ is the additional attenuation due to atmospheric phenomena (rain, clouds, scintillation, ...), $c$ is the speed of light in vacuum, $\nu$ is the carrier frequency, $\psi$ is the realization of a gamma distributed r.v. accounting for Nakagami- $m$ fading, and $\chi_{\mathrm{S}}=P_{\mathrm{tS}} G_{\mathrm{B}, \mathrm{S}} G_{\mathrm{S}} c^{2} /\left(16 A \pi^{2} \nu^{2}\right)$. Similarly to the $\mathrm{TU}$, the $\mathrm{SU}$ relies on a contention access mechanism, thus the target communication at the SU experiences a SINR:

$$
s=\frac{p_{L}}{\sum_{l=1}^{L-1} p_{l}+\rho_{\mathrm{S}}} .
$$

A manageable estimation of SINR statistic in this scenario may be derived for the values $m=1,2$ of the Nakagami parameter. This allows the formulation of the following proposition.

Proposition 2: The CCDF of the SINR for the SU when $L$ contending BSs are present may be estimated, for $m=1,2$, as:

$$
\bar{F}_{S}^{\mathrm{SU}}(s ; L) \cong \int_{s \rho_{\mathrm{S}}}^{+\infty} V_{1}\left(p_{L}\right)\left[V_{0}\left(\frac{p_{L}-s \rho_{\mathrm{S}}}{s H_{L-1}}\right)+1\right]^{L-1} \mathrm{~d} x
$$

where:

$$
\begin{aligned}
V_{j}(x)= & \frac{(-1)^{j}}{2^{2 m+j-1} \zeta x^{j}} \sqrt{\frac{\chi_{\mathrm{S}}^{\prime}}{x}} \sum_{i=1}^{2}(-1)^{i}\left\{\sqrt { \pi } \left[(2 \varepsilon x+1)^{m+j-1}\right.\right. \\
& +2(m-1)] e^{-\varepsilon x} \operatorname{erf}\left[\sqrt{\left(\bar{\varsigma}_{i}-\varepsilon\right) x}\right]-2[(m-1) \\
& \left.\cdot\left(4 \bar{\varsigma}_{i}^{2} x^{2}+2 \varepsilon x+1\right)^{j}+2(m-m j+2 j-1) \bar{\varsigma}_{i} x+m\right] \\
& \left.\cdot e^{-\bar{\varsigma}_{i} x} \sqrt{\left(\bar{\varsigma}_{i}-\varepsilon\right) x}\right\}-(-1)^{j} \\
& \cdot\left[\bar{\varsigma}_{1}^{j}\left(\bar{\varsigma}_{1} x\right)^{m-1}-(m-1)(j-1)\right] e^{-\bar{\varsigma}_{1} x}
\end{aligned}
$$

in which $\chi_{\mathrm{S}}^{\prime}=m \chi_{\mathrm{S}} /\left[\bar{\eta}_{2} R_{\mathrm{E}}\left(R_{\mathrm{E}}+h\right)\right], \varepsilon=m\left[h^{2}+2 R_{\mathrm{E}}(1-\right.$ $\left.\left.\bar{\eta}_{2}\right)\left(R_{\mathrm{E}}+h\right)\right] / \chi_{\mathrm{S}}, \bar{\varsigma}_{i}=m\left[\bar{\eta}_{i}\left(R_{\mathrm{E}}+h\right)-R_{\mathrm{E}}\right]^{2} /\left(\chi_{\mathrm{S}} \sin ^{2} \bar{\theta}_{i}\right)$ for $i=1,2$, and $\operatorname{erf}(\cdot)$ is the error function.

\section{Proof: See Appendix B.}

Also in this case, one may infer the SINR distribution for the SD from Proposition 2. In fact, the absence of interference due to the adoption of the DAMA protocol and the terrestrial location of the BS receiver imply that the CCDF of the SINR for the SD may be evaluated replacing $\rho_{\mathrm{S}}$ by $\rho_{\mathrm{E}}$ in (13) for $L=1$, thus obtaining (after integration):

$$
\bar{F}_{S}^{\mathrm{SD}}(s) \cong-V_{0}\left(s \rho_{\mathrm{E}}\right) \text {. }
$$

To clarify the results provided by Propositions 1 and 2, two sets of SINR CCDFs are presented by adopting the PHY layer parameters in Table I. These values, which will be used throughout the paper, are selected according to recent channel measurements at $28 \mathrm{GHz}$ for the 5G links [33], and to operative frequency plans of LEO satellites working in the K-band for the mmWave space links [34]. The first set of results refers to the TD and the TU for different $N$ values (Fig. 3(a)), while the second set refers to the SD and the SU for different $L$ values (Fig. 3(b)). All theoretical curves (lines) are validated by independent Monte Carlo simulations (markers) to check the accuracy of the analysis. Fig. 3(a) shows that, in the considered scenario, the terrestrial links are interferencelimited, since the CCDFs of the SINR result substantially coincident and very close to one at the TD and at the TU for $N=1$. This is due, on one hand, to the small radius of the 5G cells (not larger than a few hundred of meters), which implies short communication distances, and, on the other hand, to the LOS conditions, which imply that the received power is always sufficiently higher than the noise. Both interference and noise instead affect the space links, since the communication distances are necessarily longer. As expected, the noise has a stronger impact on the SD, since the BS antenna temperature is larger than that at an nSAT. 


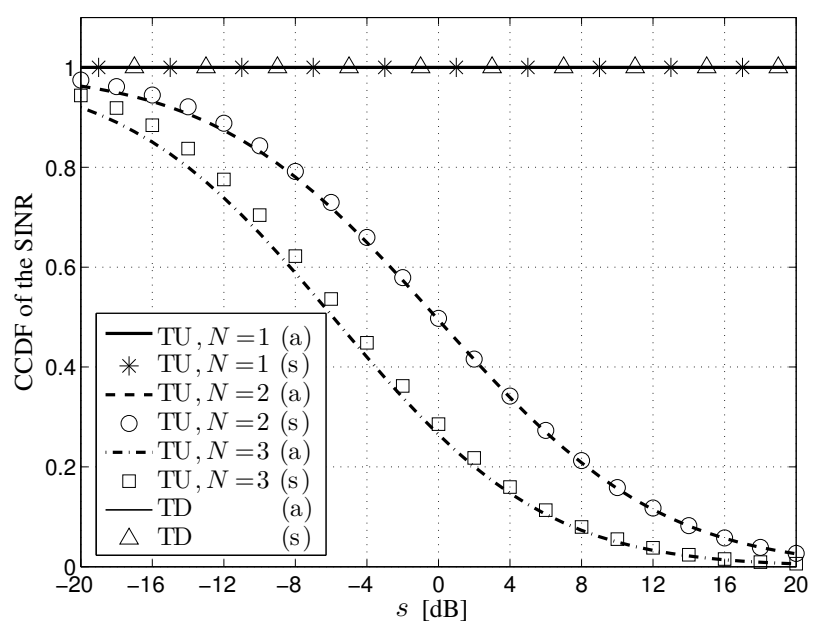

(a)

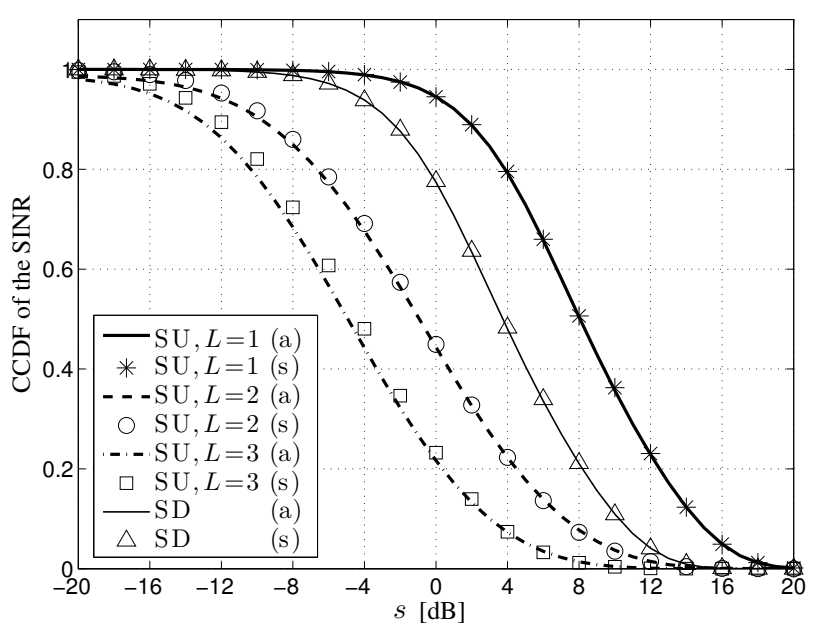

(b)

Fig. 3. CCDFs of the SINR: (a) terrestrial links, (b) space links (a: analysis, s: simulation).

As a final remark, it is interesting to observe that, even if (7)-(9) and (13)-(15) may seem formally elaborated, they have the considerable advantage of providing the SINR distribution in the presence of several physical phenomena by a unique numerical integration. This computationally cheap approach allows not only the investigation of the PHY layer performance of an integrated nSAT-5G system, but also a realistic modeling of the evolution of the access schemes implemented at the MAC layer, whose description is the aim of the next section.

\section{MAC LAYER}

From a MAC layer perspective, the use of DAMA for the downlink communications implies that the reception at the TD and SD directly depends on the propagation conditions. The usage of CRDSA at the SU and of CSA at the TU, both adopting IC to constructively exploit the collisions, requires instead to jointly consider propagation and interference for establishing the result of a transmission [15], [17]. For both schemes, the conventional performance estimation relies on the assumptions that an uncollided transmission is received and that the collided ones do not provide any information unless they are successively cleaned by IC. In practice, instead, the combined effect of noise and signal fluctuation might determine the failure of an uncollided transmission, while the capture effect might enable the reception of some transmissions even if they remain collided. Therefore, a reliable evaluation of the MAC layer performance has to account for the capture event, whose occurrence does not require the implementation of novel functionalities, since the possible capture of a not too interfered transmission is an intrinsic capability of any receiver. Accordingly, the evolution of CSA and CRDSA may be reconsidered by jointly accounting for capture and IC. To this aim, some preliminary assumptions are adopted.

Firstly, even if CSA and CRDSA may be applied to a multifrequency time division multiple access context, the discussion is developed focusing on a single carrier for simplicity [15]. Secondly, concerning both TU and SU, the timeline is subdivided in frames of $M$ slots, where each slot has a duration equal to the transmission time of a packet/bundle. A contending source (MS or BS), which is assumed frame- and slot-synchronous [15], [17], attempts the transmission of at most one packet/bundle in the respective frame. Thirdly, IC is assumed ideal, since the residual interference after IC does not significantly affect the CSA and CRDSA performance [15], [17]. The success probability for a transmission is established adopting a threshold that takes into account the modulation and the channel coding rate. More precisely, for CRDSA and downlink communications, the threshold is given by the required SINR $s$. Since (9) and (15) exactly represent the probabilities that the SINR be larger than $s$, the success probabilities at the TD and SD, which rely on a demand assignment, are immediately given by $C^{\mathrm{TD}}(s)=\bar{F}_{S}^{\mathrm{TD}}(s)$ and $C^{\mathrm{SD}}(s)=\bar{F}_{S}^{\mathrm{SD}}(s)$, respectively. For CSA, which involves packet correcting codes, the threshold is established by considering the average information rate [7]. According to these assumptions, the next subsections revisit the evolution of CSA and CRDSA by considering the combined effect of capture and IC, in order to clarify their implementation in the designed DTN architecture.

\section{A. Terrestrial uplink}

In CSA, each packet is split into $K_{\mathrm{d}}$ data segments of the same length that are encoded to produce $K$ segments still of the same length as the data ones. At each transmission, the $\left(K, K_{\mathrm{d}}\right)$ code is randomly selected within a set $\mathcal{C}$ of possible codes according to a given probability mass function (pmf) $f_{\mathcal{C}}(K)$ [17]. Besides, each slot is subdivided into $K_{\mathrm{d}}$ slices, each having a duration equal to the transmission time of a segment. The basic idea is to exploit the reception of at least $K_{\mathrm{d}}$ uncollided segments to decode the packet and cancel its remaining segments, thus enabling the iterative reception of further packets whose segments were initially collided. A possible example of TU random access frame (RAF) obtained assuming $K_{\mathrm{d}}=2$ is reported in Fig. 4 , where $\mathcal{S}_{n, k}$ denotes the 


$$
\begin{gathered}
C^{\mathrm{TU}}\left(\bar{\lambda} ; N_{1}, \ldots, N_{K}\right) \cong \int_{0}^{+\infty} \ldots \int_{0}^{+\infty} \bar{F}_{S}^{\mathrm{TU}}\left\{\left[\frac{1}{a_{1}^{\prime}} \log \left(2-K \bar{\lambda}-\sum_{k=1}^{K-1} \mathcal{I}\left(s_{k}\right)\right)-a_{3}^{\prime}\right]^{\frac{1}{a_{2}}} ; N_{K}\right\} \cdot \prod_{k=1}^{K-1} f_{S}^{\mathrm{TU}}\left(s_{k} ; N_{k}\right) \mathrm{d} s_{1} \ldots \mathrm{d} s_{K-1} . \\
f_{S}^{\mathrm{TU}}(s ; N) \cong \frac{1}{2^{N} s}\left\{\delta_{N, 1} I_{1}\left(s \rho_{\mathrm{E}}\right)+(N-1) \cdot \int_{0}^{+\infty} \frac{I_{1}(x) I_{1}\left[s\left(H_{N-1} x+\rho_{\mathrm{E}}\right)\right]}{x\left[I_{0}(x)-I_{1}(x)\right]^{2-N}} \mathrm{~d} x\right\} .
\end{gathered}
$$

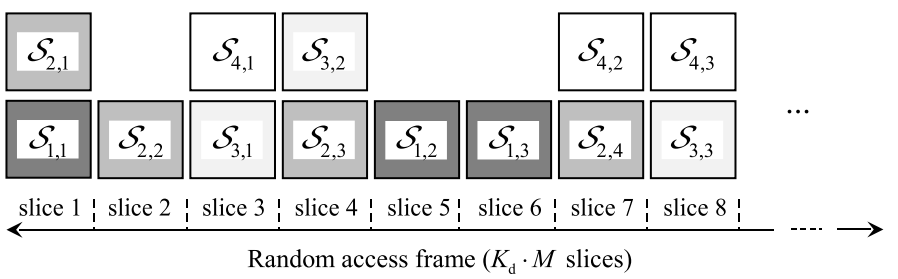

Fig. 4. TU MAC layer: possible random access frame $\left(\mathcal{S}_{n, k}\right.$ denotes the $k$-th segment of the $n$-th packet).

$k$-th segment of the $n$-th packet. In this example, the MSs 1 , 3 , and 4 adopt a code $(3,2)$, while the MS 2 adopts a code $(4,2)$. According to the CSA rules, packet 1 is received at the first iteration, since $\mathcal{S}_{1,2}$ and $\mathcal{S}_{1,3}$ are uncollided. Hence, $\mathcal{S}_{1,1}$ can be recovered and cancelled. This allows the reception of packet 2 at the second iteration from $\mathcal{S}_{2,1}$ (cleaned) and $\mathcal{S}_{2,2}$ (uncollided), and the cancellation of $\mathcal{S}_{2,3}$ and $\mathcal{S}_{2,4}$. The remaining packets 3 and 4 cannot be received, since both have two collided segments.

This evolution of CSA is based on the erasure channel, in which only two events are possible for a segment: success (all of its information available) and loss (none of its information available). The presence of capture completely changes this perspective. In an actual receiver, in fact, on one hand the propagation conditions might deteriorate an uncollided transmission, and, on the other hand, a segment involved in an unresolved collision might anyway provide a residual information. This situation can be modeled by the average information rate [7], which considers, for each packet, the SINRs $s_{1}, \ldots, s_{K}$ experienced by its $K$ segments. To this aim, rather than using the ideal, and hence optimistic, Shannon bound, which might require the introduction of upper limits on the SINR to avoid possible overestimations of the performance [36], one may more realistically consider a quadrature phase shift keying (QPSK) modulation, for which a reliable capacity expression exists [38]. Accordingly, defining the coefficients $a_{1} \cong-1.29$, $a_{2} \cong 0.93, a_{3} \cong 0.01$, and a margin $b=2.5 \mathrm{~dB}$ to account for the non-ideality of the code, the average information rate $\lambda$ may be derived by first evaluating the sequence of rates [38]:

$$
\lambda_{k} \cong \mathcal{I}\left(s_{k}\right)=2\left[1-e^{a_{1}\left(\frac{s_{k}}{2 b}\right)^{a_{2}}+a_{3}}\right], \quad k=1, \ldots, K
$$

and subsequently keeping their average value [7]:

$$
\lambda=\frac{1}{K} \sum_{k=1}^{K} \lambda_{k} .
$$

Since a modulation of order $\varsigma=4$ is employed (QPSK), in the presence of a code rate $K_{\mathrm{d}} / K$, the correct packet reception occurs if $\lambda \geq \bar{\lambda}=K_{\mathrm{d}} \log _{2} \varsigma / K=2 K_{\mathrm{d}} / K$. This concept may be combined with the SINR analysis in Subsection III-A to formulate the following proposition.

Proposition 3: The packet success probability at a TU using CSA with ideal IC and QPSK modulation when $\bar{\lambda}$ information bits per symbol are required may be estimated by (18), where $a_{1}^{\prime}=a_{1} /(2 b)^{a_{2}}, a_{3}^{\prime}=\left(a_{3}+\log 2\right) / a_{1}^{\prime}, N_{k}$ is the number of MSs that transmit in the slice containing the $k$-th segment of the packet, and the SINR probability density function (PDF) is given by (19), in which $\delta_{N, 1}$ is the Kronecker delta $\left(\delta_{N, 1}=1\right.$ if $N=1, \delta_{N, 1}=0$ otherwise).

\section{Proof: See Appendix C.}

Exploiting this proposition, the evolution of CSA in the presence of capture may be revisited. Consider again Fig. 4, but now applying (18) with $\varsigma=4$ and starting the processing of a packet when the last of its segments is arrived. At the first iteration, the first processed packet is packet 1 , for which $C^{\mathrm{TU}}(4 / 3 ; 2,1,1) \cong 0.92$. This leads, for packet 2 , to $C^{\mathrm{TU}}(1 ; 1,1,2,2) \cong 0.90$, if packet 1 is captured (that is, $\mathcal{S}_{1,1}$ is cancelled), or to $C^{\mathrm{TU}}(1 ; 2,1,2,2) \cong 0.64$, if packet 1 is not captured. This result, in turn, influences the reception of packet 3 , for which we have $C^{\mathrm{TU}}(4 / 3 ; 2,1,2) \cong$ 0.51 , if packet 2 is captured (that is, $\mathcal{S}_{2,3}$ is cancelled), or $C^{\mathrm{TU}}(4 / 3 ; 2,2,2) \cong 0.24$, if packet 2 is not captured. If at least one among packets 1,2 , and 3 is captured, a second iteration is carried out to check if other captures can occur. Otherwise, a second iteration is not necessary since no modifications of the RAF have occurred with respect to the first iteration. In general, in the receiver implementation, the capture experiment for a packet is repeated only if its success probability changes because of one or more cancellations at the previous iteration. Besides, to avoid possible unrealistic overestimations of the number of successes, a maximum of two capture experiments is allowed for each packet.

\section{B. Space uplink}

Differently from CSA, CRDSA adopts a repetition approach by transmitting two copies of a bundle in two different slots [15]. If one copy is successful, the receiver recovers and cancels the other, thus allowing the possible iterative recovery of further initially collided bundles. For example, in the case reported in Fig. 5, where $\mathcal{B}_{l, k}$ identifies the $k$-th copy of the $l$-th bundle, $\mathcal{B}_{1,2}$ is uncollided. Thus, at the first iteration, bundle 1 is received and $\mathcal{B}_{1,1}$ is cancelled. This enables to clean $\mathcal{B}_{2,1}$, and hence to receive, at the second iteration, bundle 2 and cancel $\mathcal{B}_{2,2}$. Bundles 3 and 4 are not received, 


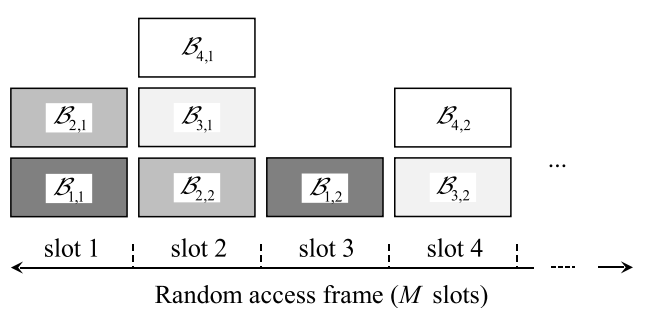

Fig. 5. SU MAC layer: possible random access frame $\left(\mathcal{B}_{l, k}\right.$ identifies the $k$-th copy of the $l$-th bundle).

since their copies are involved in unresolved collisions. Also in this case, the capture event may modify this evolution. Since, in CRDSA, no packet correcting codes are adopted, the success probability for a copy transmitted in a slot is directly given by (13), thus $C^{\mathrm{SU}}\left(s ; L_{\mathrm{B}}\right)=\bar{F}_{S}^{\mathrm{SU}}\left(s ; L_{\mathrm{B}}\right)$, where $s$ is the SINR threshold and $L_{\mathrm{B}}\left(0 \leq L_{\mathrm{B}} \leq L\right)$ is the number of BSs that have transmitted in that slot. Consider again Fig. 5 with $s=2.23 \mathrm{~dB}(\cong 5 / 3)$ [39, Tab. 13], for which the corresponding success probabilities can be directly inferred from Fig. 3(b) as $C^{\mathrm{SU}}(5 / 3 ; 1) \cong 0.88, C^{\mathrm{SU}}(5 / 3 ; 2) \cong 0.31$, $C^{\mathrm{SU}}(5 / 3 ; 3) \cong 0.13$. At each iteration, the receiver performs at most one capture experiment per slot by sequentially moving from the first one. Thus, at the first iteration, $\mathcal{B}_{1,1}$ is received with a $31 \%$ probability, $\mathcal{B}_{2,2}$ with a $13 \%$ probability, $\mathcal{B}_{1,2}$ with an $88 \%$ probability, and $\mathcal{B}_{3,2}$ with a $31 \%$ probability. Depending on the results of the capture experiments, the copies of the successful bundles are cancelled or not, and, at the second iteration, further capture experiments are carried out for the sole copies experiencing a variation of the success probability with respect to the previous iteration, with a maximum of two overall experiments per copy (consistently with the approach previously described for CSA).

To clarify the impact of the success probability on the decoding procedure, the parameters in Table II, which will be also adopted for the rest of the paper, are used for comparing the throughput of CSA and CRDSA with and without capture (Fig. 6). To obtain a fair comparison, no limits have been imposed on the number of iterations in the absence of capture. The transmission system adopted at the TU involves a QPSK modulation with two code rates generated according to the $\mathrm{pmf}$ $f_{\mathcal{C}}(K)$ [39, Tab. 13], while that at the SU involves a QPSK modulation with a fixed code rate $3 / 5$. More precisely, for CSA, the two possible thresholds on the average information rate are $\bar{\lambda}=4 / 3$ and $\bar{\lambda}=1$, corresponding to $K=3$ and $K=4$, respectively. Observe that both the threshold on each information rate at the TU and that on the SINR at the SU are coherent with these values, where, in particular, the value $3 / 5$ represents the rate of the access scheme for CSA [17], and the rate of the bundle encoder for CRDSA [39, Tab. 13].

\begin{tabular}{|l|l|}
\hline$s=2.23 \mathrm{~dB}$ & $\varsigma=4$ \\
\hline$K_{\mathrm{d}}=2$ & $f_{\mathcal{C}}(K=3)=2 / 3, f_{\mathcal{C}}(K=4)=1 / 3$ \\
\hline
\end{tabular}

TABLE II

MAC LAYER PARAMETERS [17], [39].

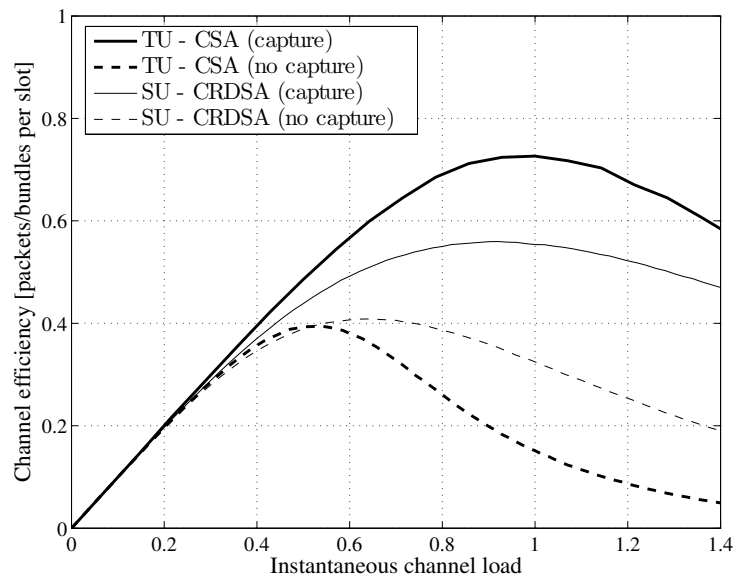

Fig. 6. Throughput as a function of the offered load for the CSA and CRDSA schemes with and without capture.

Fig. 6, which is obtained for $N=L=50$ and $M=35$, reveals that in general the capture effect provides considerable improvements for both schemes. In particular, the throughput increase in the presence of capture for CRDSA is not strictly due to an increase of the successful receptions with respect to the absence of capture, but to a positive balance between unsuccessful receptions due to the noise and successful receptions due to the captured collided replicas. This effect is also experienced by the different segments when CSA is applied in the presence of capture. However, in this case, the throughput benefits are mainly due to a combination of coded random access and SINR fluctuations, which generates a beneficial phenomenon of interference diversity among the slices containing different segments.

\section{NET AND HIGHER LAYERS}

Once the access rules are established, the subsequent functionality that must be addressed concerns the routing of the packets/bundles. As shown in Figs. 1 and 2, in the presented network architecture the routing process is split into three portions: the IP routing from $\mathrm{M}_{N}$ to the terrestrial interface of $\mathrm{B}_{L}$, the DTN routing from the satellite interface of $\mathrm{B}_{L}$ to the satellite interface of $\mathrm{B}^{\prime}$ (DTN portion of the network), and the IP routing from the terrestrial interface of $\mathrm{B}^{\prime}$ to $\mathrm{M}^{\prime}$. In the terrestrial links, the routing process is carried out by the IP protocol at the NET layer, while, in the satellite links, by the BP at the DTN layer. Routing functionalities have to be hence properly managed to allow the routing through heterogeneous sub-networks. Besides, a proper routing algorithm has to be chosen or defined for forwarding path computation through the DTN portion, depending on the available information, such as the available resources and the current or estimated status of the network.

\section{A. Routing functionality management}

Considering the different layers in charge of the routing process in the heterogeneous network portions, different aspects have to be managed to guarantee successful delivery. 
- Address translation: The MSs are identified by their IP addresses, but the IP and UDP/TCP headers are removed from the packets before sending them through satellite links, as shown in Fig. 2. However, the destination IP address is required when the packets reach their destination BS. To address this issue, we define a bundle extension block (BEB) that contains three fields: the source IP address (4 octets), the destination IP address (4 octets), and the type ( 1 octet). The source IP address guarantees the identification of the source node $M_{N}$ (mandatory also for bidirectional communications). The destination IP address ensures the data delivery over the $\mathrm{B}^{\prime}-\mathrm{M}^{\prime}$ portion. The type field discriminates between the data bundles, which contain the application data, and the status bundles, which contain the nSAT buffer occupancy information exploited for the DTN routing process.

- Packet encapsulation: Additional functionalities related to the DTN paradigm have to be included to allow the packet transmission through the space segment. To this aim, an additional header has to be attached to each packet traversing the DTN network portion. More precisely, $\mathrm{M}_{N}$ sends its data packets to $\mathrm{B}_{L} \cdot \mathrm{B}_{L}$ encapsulates the received data packets in data bundles then adding the standard primary bundle header [25], and the defined BEB. $\mathrm{B}^{\prime}$ de-encapsulates the received data bundles, reads the destination IP address stored in the $\mathrm{BEB}$, and sends the extracted data packets to $\mathrm{M}^{\prime}$.

- Route discovery: Networks with dynamic topology changes require a route discovery mechanism. However, in the considered network, the topology normally changes in a predictable way, because the satellites are the only mobile nodes and change their position within predefined orbits. The route computation is therefore achievable by defining the subsequent topology changes as network "snapshots", each of them identifying a different network topology and its duration.

Within the DTN network portion, the BEB's type field allows a BS to perform the routing decision considering also the number of bundles stored in the nSAT buffer. To enable this capability, an extension of the widely adopted CGR algorithm is here presented.

\section{B. Buffer aware contact graph routing (BA-CGR) algorithm}

The CGR algorithm is a typical solution to solve end-toend bundle routing in satellite DTN networks [40]. It relies on the exploitation of the full information about future contacts. CGR is specifically designed for use in networks where the changes in connectivity are planned and scheduled rather than predicted or discovered. To this aim, a global contact plan (CP) is built and distributed in advance to all DTN nodes. The CP represents a time-ordered list of scheduled contacts, in which each event is an assertion that a transmission between a node pair at a certain nominal transmission rate will begin and end at fixed times [26]. This assertion implicitly defines

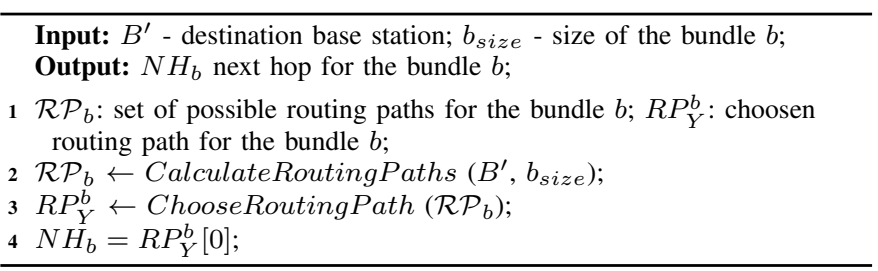

(a) BA-CGR algorithm

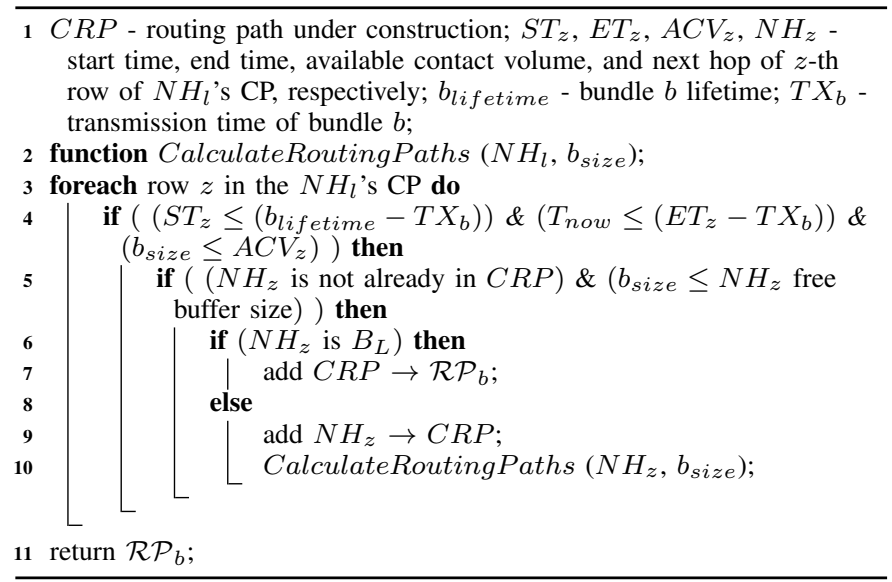

(b) Function CalculateRoutingPaths

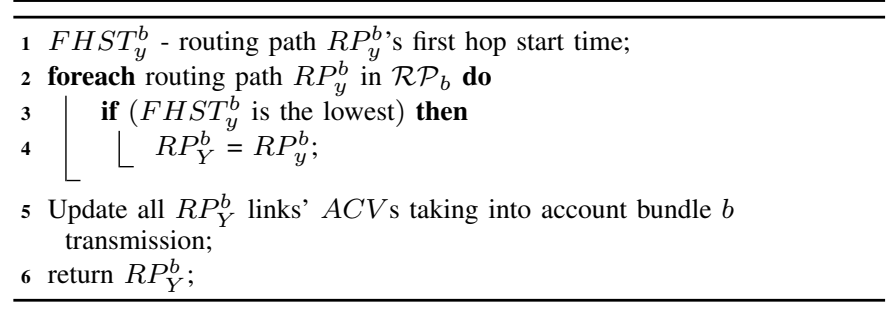

(c) Function ChooseRoutingPath

Fig. 7. BA-CGR algorithm.

the volume of the contact, that is, the maximum amount of data that can be transferred during the contact. Given a $\mathrm{CP}$, each DTN node locally constructs a contact graph data structure, which contains, for each other node of the network, a list of objects. Each object encapsulates start time, end time, and data transmission rate of each contact. A route for a given bundle is defined as a sequence of contacts such that: the first contact is from the bundle's source node to some other node, the subsequent contacts are from the receiving node of the previous contact to some other node, and the last contact is from some node to the bundle's destination node, without loops. In the original version of CGR, each intermediate node calculates its own graph and makes its own decision by minimizing the bundle delivery time, even if other metrics have been proposed [40]. The here proposed BA-CGR considers, beside the bundle delivery time, the nSAT buffer occupancy, in order to manage a heterogeneous DTN scenario characterized by long latencies and possible losses.

In the BA-CGR algorithm, each data bundle $b$ (type $=0$ ) may be scheduled for upload by $\mathrm{B}_{L}$ considering an already or forthcoming visible nSAT. In particular, to reduce both the delivery time and the possible losses due to buffer overflow, 
$\mathrm{B}_{L}$ selects an nSAT $S_{q}$ for the upload of $b$ by jointly exploiting the known a priori information about the nSATs' movements, that is, the timing information about the contacts between the nSATs and the BSs, and the dynamically changing information about the nSAT buffer occupancy. The details of this selection are reported in Fig. 7 and explained in the following. Through the $\mathrm{CP}$ stored inside its memory, $\mathrm{B}_{L}$ is aware of all contacts between the BSs and the nSATs during all network lifetime, including start times $S T s$, end times ETs, and available contact volumes $A C V s$ (Fig. 7(b)). Exploiting this information, the $\mathrm{BS} \mathrm{B}_{L}$ calls the function CalculateRoutingPaths, which scans the $\mathrm{B}_{L}$ 's $\mathrm{CP}$ and computes each possible path $R P_{y}^{b}$ between $\mathrm{B}_{L}$ and $\mathrm{B}^{\prime}$, i.e., it computes all pairs of links $\mathrm{B}_{L}-\mathrm{S}_{q}$ and $\mathrm{S}_{q}-\mathrm{B}^{\prime}$ for $q=1, \ldots, Q$, that can be used to send $b$ from $\mathrm{B}_{L}$ to $\mathrm{B}^{\prime}$. The computation starts from the last hop, i.e., $\mathrm{B}^{\prime}$, and proceeds backward to $\mathrm{B}_{L} \cdot \mathrm{B}_{L}-\mathrm{S}_{q}$ link is exploitable if it is not ended yet and its $A C V$ is greater than the size $b_{\text {size }}$ of the bundle $b$. The $\mathrm{S}_{q}-\mathrm{B}^{\prime}$ link is exploitable if its end time is higher than the transmission time $T X_{b}$ of $b$ through the $\mathrm{B}_{L}-\mathrm{S}_{q}$

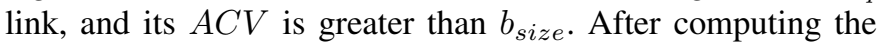
complete set of possible paths $\mathcal{R} \mathcal{P}_{b}$ for $b, \mathrm{~B}_{L}$ calls the function ChooseRoutingPath to choose the path $R P_{Y}^{b}$ that allows $b$ to reach $B^{\prime}$ with the lowest delivery time.

Once the nSAT selection is complete, the $\mathrm{CP}$ of $\mathrm{B}_{L}$ is consequently updated. Observe that the start and end contact times are fixed and a priori known, but the nSAT buffer occupancies change depending on traffic flows. Therefore, the nSAT periodically sends a bundle to $\mathrm{B}_{L}$, called status bundle (type $=1$ ), which contains information about the amount of data stored inside the nSAT buffer for each destination BS, in order to make $\mathrm{B}_{L}$ aware of the current value and trend of the the nSAT free storage space. The periodic update is required in case of multiple simultaneous transmissions from multiple BSs. $B_{L}$, in fact, does not know if there are other BSs that are transmitting to the same nSAT, so $\mathrm{B}_{L}$ cannot exactly know how the nSAT free buffer space evolves during the contact. Periodical updates of the nSAT buffer status allow $\mathrm{B}_{L}$ to adjust the amount of data it can still send. The propagation delay of the satellite links is also taken into account by considering the most conservative case for the nSAT buffer space evolution, i.e., the nSAT receives the maximum amount of data during the satellite propagation time. By this improvement, BA-CGR enables data exchange among $5 \mathrm{G}$ MSs located in different cells through an nSAT constellation, accounting for delivery time reduction and buffer overflow avoidance. The advantages and the performance provided by this novel routing algorithm are discussed in detail in the next section.

\section{RESULTS}

\section{A. Simulation platform}

To estimate the performance of BA-CGR, the entire network architecture is implemented in a developed discrete-time discrete-event simulator based on the combination of Matlab with NS3 [41], so as to obtain an integrated Matlab-NS3 platform consisting of four main modules.

The first module (NS3 code) identifies the configuration parameters, that is, the simulation duration $\tau_{\text {sim }}$, the nominal

\begin{tabular}{|l|l|l|l|}
\hline$\tau_{\text {sim }}=24 \mathrm{hrs}$ & $\tau_{\mathrm{B}}=1 \mathrm{hr}$ & $\varrho_{\mathrm{B}}=1000$ & $\varrho_{\mathrm{P}}=1250$ bytes \\
\hline$O=4$ & $\beta=535 \mathrm{Mbits} / \mathrm{s}$ & $M=10$ & $\varpi=4$ \\
\hline
\end{tabular}

TABLE III

SYSTEM PARAMETERS.

bit rate $\beta$, the number $N$ of MSs per cell, the number $L$ of BSs visible to an nSAT, the number $Q$ of nSATs, the number $O$ of orbital planes, the latitude, longitude, altitude (LLA) coordinates of each BS, the orbital parameters, and the buffer size $B_{\text {size }}$ of each nSAT. The nSAT positions are computed and updated through a widespread orbital model called NORAD SGP4 [42], which takes as input the current time and the standard two-line orbital element set of each satellite to give as output its ECI position and velocity in agreement with the geodesic parameters of the Earth. In the simulated scenario, all nSATs are uniformly distributed among circular orbits and equally spaced within each orbit. Both nSAT ECI and BS LLA positions are converted in ECEF coordinates to compute the $\mathrm{CP}$ for the BA-CGR algorithm.

The second module (NS3 code) defines the number of traffic flows and their characteristics. To properly check the introduced functionalities, the considered end-to-end communications involve MS pairs located in $5 \mathrm{G}$ cells not simultaneously visible to the same nSAT. Terrestrial traffic flows can be modeled by different statistical processes, such as Poisson, on/off, and Markov modulated Poisson ones [43], depending on the specific application (web traffic, video streaming, email, ...). Considering that the proposed nSAT-5G architecture is designed for delay-tolerant data transport services, each MS is assumed to generate traffic following an on/off process with constant inter-period time $\tau_{\mathrm{B}}$, fixed number of packets $\varrho_{\mathrm{B}}$ transmitted per active period, and given packet size $\varrho_{\mathrm{P}}$.

The third module (Matlab code) is the discrete-time MAC/PHY module, which implements the DAMA, CSA, and CRDSA access schemes described in Section IV and the SINR statistics derived in Section III. The time-based approach is adopted to jointly consider IC and capture during the analysis of the received RAF of $M$ slots. Besides, a retry limit $\varpi$ is introduced to improve the reliability of the access procedure. Thus, if a packet/bundle is not correctly received in a RAF, its retransmission is allowed in the subsequent RAF, for a maximum of $\varpi$ times.

The fourth module (NS3 code) implements the DTN architecture including the BA-CGR algorithm with the BEB presented in Section V. This module calls the MAC/PHY one through a specific script once the links between the source BS and the chosen nSAT, and between the chosen nSAT and the destination BS, are active (i.e., when an nSAT becomes visible to a $\mathrm{BS})$.

\section{B. Performance evaluation}

The designed nSAT-5G mmWave architecture is applied to a data transport scenario characterized by the parameters shown in Table III. The nominal bit rate $\beta=\beta_{\mathrm{e}} B_{\mathrm{W}} \cong 535 \mathrm{Mbits} / \mathrm{s}$ is selected according to the adopted $3 / 5-\mathrm{QPSK}$ code-modulation 


\begin{tabular}{|c|c||c|c||c|c||c|c|c|}
\cline { 4 - 9 } \multicolumn{2}{c|}{} & \multicolumn{2}{c||}{$\begin{array}{c}\text { Throughput } \\
{[\text { Mbits/s] }}\end{array}$} & \multicolumn{2}{c||}{ Delay [s] } & \multicolumn{3}{c|}{ Loss rate [\%] } \\
\hline$N$ & $L$ & TU & SU & TU & SU & TU & SU & $\begin{array}{c}\text { Buffer } \\
\text { overflow }\end{array}$ \\
\hline \hline 5 & 5 & 133.06 & 126.09 & 0.01 & 0.06 & 0.00 & 0.00 & 0.00 \\
\hline \hline 10 & 5 & 258.88 & 126.23 & 0.01 & 0.12 & 0.00 & 0.00 & 0.00 \\
\hline 15 & 5 & 355.62 & 126.31 & 0.01 & 0.19 & 0.00 & 0.00 & 0.00 \\
\hline 20 & 5 & 389.72 & 126.25 & 0.01 & 0.26 & 0.41 & 0.05 & 0.00 \\
\hline \hline 5 & 10 & 133.00 & 224.99 & 0.01 & 0.07 & 0.00 & 0.05 & 0.00 \\
\hline 5 & 15 & 133.03 & 275.27 & 0.01 & 0.09 & 0.00 & 0.91 & 0.00 \\
\hline 5 & 20 & 133.03 & 280.43 & 0.01 & 0.11 & 0.00 & 4.94 & 0.00 \\
\hline
\end{tabular}

TABLE IV

PERFORMANCE AS A FUNCTION OF THE NUMBER OF MSS PER CELL AND BSS VISIBLE TO AN NSAT FOR $Q=60$ AND $B_{\text {Size }}=1$ GBYTE (CGR AND BA-CGR PROVIDE THE SAME RESULTS FOR THIS SET OF TESTS).

pair, where $\beta_{\mathrm{e}} \cong 1.19 \mathrm{bits} /(\mathrm{s} \cdot \mathrm{Hz})$ is the spectral efficiency [39, Tab. 13], and $B_{\mathrm{W}}$ is the available bandwidth (Table I).

The first set of results in Table IV investigates the throughput, delay, and loss rate at the TU and SU as a function of $N$ and $L$ for $Q=60$ and $B_{\text {size }}=1$ Gbyte, which is sufficiently high to avoid buffer overflow (in fact, in this case CGR and BA-CGR provide the same performance). The table shows that, for a fixed number of BSs (MSs), the increase of $N(L)$ leads to an increase of the throughput at the TU (SU). Besides, if the objective is to support a given number of terrestrial traffic flows, i.e., to operate with a fixed $N \times L$ product, the selection of a lower $L$ value and a higher $N$ one is preferable in terms of reliability. This may be noticed by comparing, for example, the overall loss rate at both the TU and SU for the cases $20 \times 5$ and $5 \times 20$. The opposite choice should be made when the objective is the reduction of the delay, which represents one of the benefits provided by the $5 \mathrm{G}$ BS densification strategy for an integrated 5G-nSAT network.

The second set of simulations compares the CGR and BACGR algorithms considering $N=L=20, Q=60$, and different values of the nSAT buffer size (Table V). In this case, to outline the effects of $B_{\text {size }}$, the table distinguishes not only between MAC/PHY and buffer losses, but also between TU/SU access delay and waiting time at the source BS. The results show that, using the BA-CGR algorithm, no buffer losses occur, regardless of the value of $B_{\text {size }}$. Conversely, focusing on the results obtained for $B_{\text {size }}=250$ and 125

\begin{tabular}{|c||c|c||c|c||c|c||c|c|}
\cline { 2 - 8 } \multicolumn{1}{c|}{} & \multicolumn{4}{c||}{ Delay [s] } & \multicolumn{4}{c|}{ Loss rate [\%] } \\
\cline { 2 - 9 } \multicolumn{1}{c|}{} & $\begin{array}{c}\text { TU/SU } \\
\text { access }\end{array}$ & \multicolumn{1}{|c||}{$\begin{array}{c}\text { Waiting time } \\
\text { at BS }\end{array}$} & \multicolumn{2}{c|}{$\begin{array}{c}\text { TU/SU } \\
\text { access }\end{array}$} & \multicolumn{2}{c|}{$\begin{array}{c}\text { Buffer } \\
\text { overflow }\end{array}$} \\
\hline $\begin{array}{c}B_{\text {size }} \\
\text { [Mbytes] }\end{array}$ & CGR & $\begin{array}{c}\text { BA- } \\
\text { CGR }\end{array}$ & CGR & $\begin{array}{c}\text { BA- } \\
\text { CGR }\end{array}$ & CGR & $\begin{array}{c}\text { BA- } \\
\text { CGR }\end{array}$ & CGR & $\begin{array}{c}\text { BA- } \\
\text { CGR }\end{array}$ \\
\hline \hline 1000 & 0.35 & 0.35 & 0.00 & 0.00 & 7.09 & 7.09 & 0.00 & 0.00 \\
\hline 500 & 0.35 & 0.35 & 0.00 & 0.00 & 7.09 & 7.09 & 0.00 & 0.00 \\
\hline 250 & 0.19 & 0.21 & 0.00 & 417.24 & 6.16 & 7.07 & 45.75 & 0.00 \\
\hline 125 & 0.10 & 0.21 & 0.00 & 834.13 & 5.80 & 7.08 & 70.17 & 0.00 \\
\hline
\end{tabular}

TABLE V

DELAY AND LOSS RATE AS A FUNCTION OF THE NSAT BUFFER SIZE FOR $N=L=20$ AND $Q=60$

\begin{tabular}{|c||c|c||c|c|}
\cline { 2 - 5 } \multicolumn{1}{c||}{} & \multicolumn{2}{c||}{$B_{\text {size }}=250$ Mbytes } & \multicolumn{2}{c|}{$B_{\text {size }}=125$ Mbytes } \\
\hline$Q$ & $\begin{array}{c}\text { Waiting time } \\
\text { at BS [s] }\end{array}$ & $\begin{array}{c}\text { TU/SU access } \\
\text { loss rate [\%] }\end{array}$ & $\begin{array}{c}\text { Waiting time } \\
\text { at BS [s] }\end{array}$ & $\begin{array}{c}\text { TU/SU access } \\
\text { loss rate [\%] }\end{array}$ \\
\hline \hline 60 & 417.24 & 7.07 & 834.13 & 7.08 \\
\hline 120 & 230.69 & 7.08 & 249.07 & 7.04 \\
\hline 240 & 161.10 & 7.14 & 193.85 & 6.99 \\
\hline 480 & 129.85 & 7.04 & 162.74 & 7.02 \\
\hline
\end{tabular}

TABLE VI

BA-CGR PERFORMANCE AS A FUNCTION OF THE NSAT NUMBER FOR $N=L=20$.

Mbytes, the usage of the conventional CGR algorithm leads to not negligible loss rates when the size of the nSAT buffer is reduced, up until the achievement of unacceptable values for any application having minimum reliability requirements. The reduced loss rate due to buffer overflow provided by the BA-CGR is achieved at the expense of a higher waiting time at the BS, which, however, for non-delay-sensitive services, represents a minor concern with respect to the control of the losses.

To reduce the delay, two possible approaches involving the BA-CGR algorithm are discussed. The first one, conceptually more immediate, consists in increasing the number of nSATs. In this situation, the results in Table VI show that the reduction of the waiting time at the BS is evident, owing to the larger upload capacity of the constellations (i.e., to the larger amount of opportunities that the BSs have to upload the data stored in their buffers). This solution offers a lower delay without affecting the loss rate, whose values are comparable to those reported in the first row of Table V. However, this solution has a considerable drawback: a higher production cost of the overall network. For this reason, a second approach to reduce the delay is proposed. This approach, which is suitable for loss-tolerant services, consists in introducing a parameter $\varphi$ in the BA-CGR algorithm to dynamically set the amount of data that a source BS is allowed to drop without trying to send it. This action may be reasonable when a source BS has to manage a traffic flow with a deadline time and the visible nSAT has not enough buffer space for uploading all the data. The allowance of dropping the packets that have no chance to arrive in time may be suitable, for example, for streaming applications adopting scalable video encoders, in which the encoded packets can be prioritized according to their contribution to the final video quality. For the sake of simplicity, in the presented example, $\varphi$ is set as a percentage of $B_{\text {size }}$, but, if desired, more sophisticated choices might be considered, such as selecting the $\varphi$ value in agreement with the performance requirement of each traffic flow.

Fig. 8 reports, for $N=L=20$, the waiting time at the BS and the SU loss+dropping rate as a function of $\varphi$, considering different buffer sizes and number of nSATs. Note that, since no unwanted buffer losses occur in BACGR, the SU loss+dropping rate identifies the losses due to the MAC/PHY procedures and to the a-priori $\varphi$-dropping. In detail, the results for the lowest value of $\varphi(\varphi=5 \%)$ in Fig. 8 are close to those reported in the last two rows of Table V, 


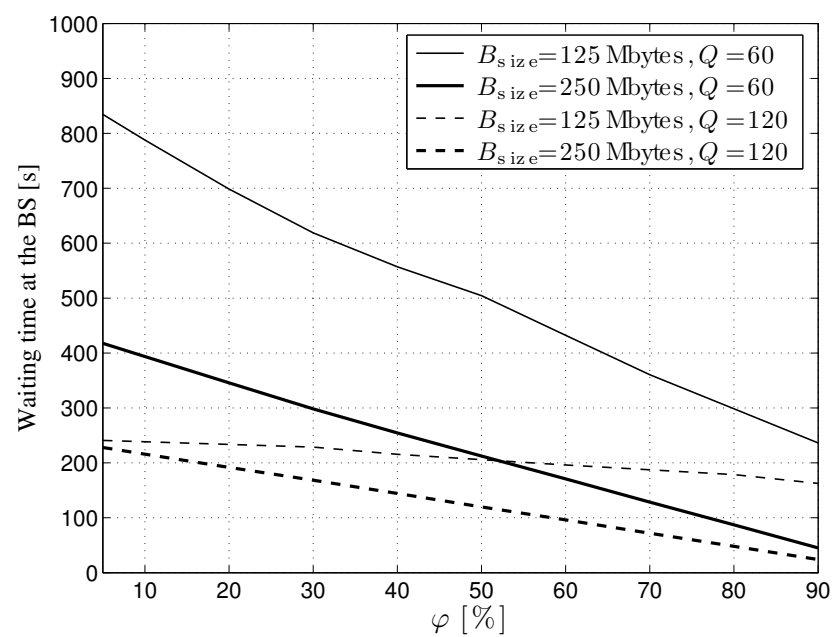

(a)

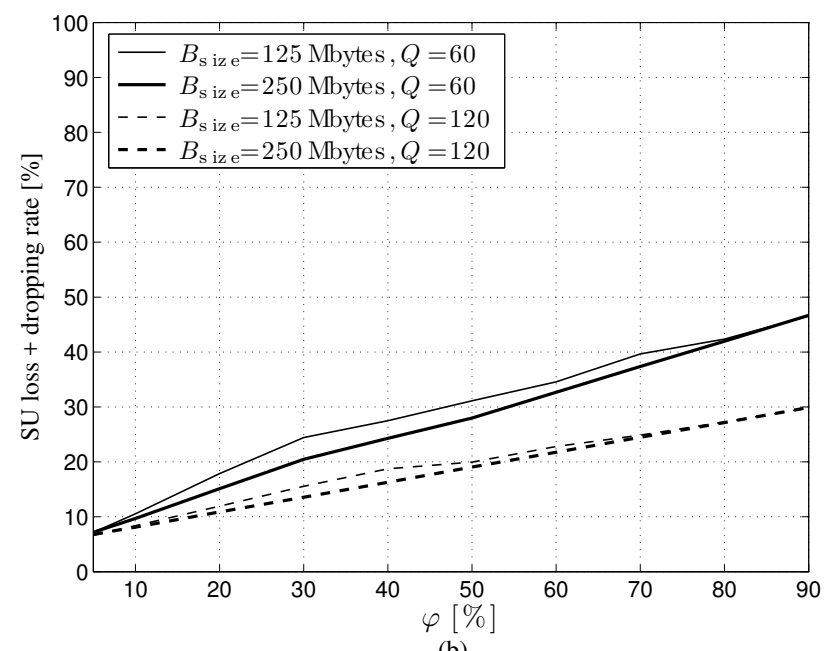

(b)

Fig. 8. BA-CGR performance as a function of $\varphi$ for $N=L=20$ : (a) waiting time at the BS, (b) SU loss+dropping rate.

corresponding to the case $\varphi=0$. By increasing $\varphi$, the amount of packets sent through the SU decreases, thus reducing the number of required nSAT contacts and, in turn, the waiting time at the source BS. It is worth to observe that, even if the loss/delay trend for BA-CGR with $\varphi \rightarrow 1$ would approach that for CGR, the two algorithms do not actually operate in the same way. For equal loss/delay performance, CGR sends all the waiting packets and the portion of them which exceeds the nSAT buffer space is lost due to overflow, while BA-CGR maintains the advantage of dropping the packets before their transmission at the SU, potentially allowing the BS to select which packets can be dropped. A joint observation of Table VI for $Q=240,480$ and Fig. 8 for $Q=120$ finally reveals that the $\varphi$-dropping technique may represent a useful trade-off between reliability and complexity to obtain delay reductions without significantly increasing the constellation size.

\section{Inter-satellite links (ISLs)}

The main impact on the end-to-end delivery time is due to the time required by the satellite to reach the destination, because, when an nSAT performs an upload from a source $\mathrm{BS}$, the packets remain stored in the nSAT buffer until the contact with the destination BS. A reduction of this quantity, and also, indirectly, of the waiting time at the source BS, may be achieved by implementing inter-satellite communication. In this case, the routing path may involve more nSATs, which can exchange the packets for a faster final delivery. This possibility is here explored by considering intra-orbit ISLs, that is, allowing the packet exchange between each nSAT and its previous and following ones lying in the same orbital plane.

The results in Table VII present the average delivery time (ADT), i.e., the average time required to complete the endto-end communication, as a function of $N$ and $L$ with and without ISLs for $Q=720$ and $B_{\text {size }}=1$ Gbyte (similarly to Table IV, also in this case CGR and BA-CGR provide the same performance). The value $Q=720$ is selected to guarantee a distance between adjacent nSATs sufficient to

\begin{tabular}{|c|c||c|c|}
\hline$N$ & $L$ & ADT (without ISL) [s] & ADT (with ISL) [s] \\
\hline \hline 5 & 5 & 351.63 & 64.39 \\
\hline \hline 10 & 5 & 353.37 & 66.03 \\
\hline 15 & 5 & 354.96 & 67.72 \\
\hline 20 & 5 & 356.50 & 69.26 \\
\hline \hline 5 & 10 & 353.62 & 66.38 \\
\hline 5 & 15 & 354.87 & 67.33 \\
\hline 5 & 20 & 356.24 & 69.00 \\
\hline
\end{tabular}

TABLE VII

ADT AS A FUNCTION OF THE NUMBER OF MSS PER CELL AND BSS VISIBLE TO AN NSAT WITH AND WITHOUT ISLS FOR $Q=720$ AND $B_{\text {size }}=1$ GBYTE (CGR AND BA-CGR PROVIDE THE SAME PERFORMANCE).

achieve a SINR $s=2.23 \mathrm{~dB}$ (Table II) for an orbit altitude $h=400 \mathrm{~km}$ (Table I) in LOS conditions. The main reason for the considerable ADT decrease in the presence of ISLs is due to the fact that, in some situations, a couple of nSATs belonging to the same orbital plane are one in contact with the source BS and the other with the destination BS. There is hence an end-to-end path between source and destination MSs where all links (space and terrestrial) are active at the same time. This situation, which allows the nSATs to deliver the received packets when the contact is still active, also leads to an increase of the uploaded data per contact. Of course, this represents the most favorable situation for using ISLs, which, in the worst cases, may be even not exploited, such as when the Earth rotation makes their usage not advantageous. However, in general, the benefits of ISLs are considerable, as it is confirmed by Table VIII, which compares the CGR and BA-CGR algorithms for $N=L=20$, considering different number of satellites and different values of the nSAT buffer size. With respect to the case $Q=720$, the use of $Q=360$ nSATs implies a higher distance between the adjacent ones, which leads to a SINR equal to $-2.35 \mathrm{~dB}$. This value requires the usage of a 1/4-QPSK code-modulation pair providing a spectral efficiency of 0.49 bits/(s.Hz) [39, Tab. 13] and, in turn, 


\begin{tabular}{|c||c|c||c|c||c|c||c|c|}
\cline { 2 - 9 } \multicolumn{1}{c|}{} & \multicolumn{4}{c||}{$Q=720$} & \multicolumn{4}{c|}{$Q=360$} \\
\cline { 2 - 9 } \multicolumn{1}{c|}{} & \multicolumn{2}{c||}{ ADT [s] } & \multicolumn{1}{c||}{ Loss rate [\%] } & \multicolumn{2}{c|}{ ADT [s] } & \multicolumn{2}{c|}{ Loss rate [\%] } \\
\hline$B_{\text {size }}$ [Mbytes] & CGR & BA-CGR & CGR & BA-CGR & CGR & BA-CGR & CGR & BA-CGR \\
\hline \hline 1000 & 73.48 & 73.48 & 7.09 & 7.09 & 159.42 & 159.42 & 6.92 & 6.92 \\
\hline 500 & 73.09 & 73.09 & 7.09 & 7.09 & 159.42 & 176.96 & 6.89 & 6.89 \\
\hline 250 & 65.58 & 179.95 & 11.41 & 7.07 & 123.72 & 295.23 & 12.55 & 6.96 \\
\hline 125 & 57.12 & 209.16 & 21.94 & 7.08 & 101.01 & 343.17 & 25.89 & 6.98 \\
\hline
\end{tabular}

TABLE VIII

ADT AND LOSS RATE AS A FUNCTION OF THE NSAT NUMBER AND BUFFER SIZE WITH ISLS FOR $N=L=20$.

a nominal bit rate equal to $221 \mathrm{Mbits} / \mathrm{s}$ (lower than that so far adopted and reported in Table III).

Coherently with the previous discussion regarding Table II, also in the presence of ISLs, for a given $Q$ value, a $B_{\text {size }}$ reduction leads, for BA-CGR, to an increase of the ADT due to an increase of the waiting time at the BS, and, for CGR, to an increase of the loss rate due to buffer overflow. Concerning the impact of the constellation size on the ADT, one may notice that, as expected, the halving of the number of nSATs leads to a considerable delay increase, which, however, results proportionally more pronounced when a larger buffer is adopted. In fact, the ADT is more than doubled for both algorithms when buffers of 1000 and 500 Mbytes are adopted. Instead, for $B_{\text {size }}=250$ and 125 Mbytes, the CGR case experiences an ADT increase in the order of $80 \%$ when $Q$ is halved, while, in the same conditions, the ADT increase for BA-CGR is in the order of $60 \%$. From Table VIII, we may also notice that the loss rate is weakly influenced by the halving of $Q$ when BA-CGR is used, while, when CGR is adopted, the utilization of a smaller buffer leads to a certain increase of the losses. Of course, however, it is worth to observe that, both for CGR and BA-CGR, and regardless of the specific $Q$ and $B_{\text {size }}$ values, the enabling of inter-satellite communication implies a higher on-board computational cost, considering that in this case also the nSATs have to perform the next hop computation.

\section{CONCLUSIONS}

This paper has presented a complete network architecture for an integrated nSAT-5G mmWave system. The architecture, which combines the DTN paradigm with the advanced CSA and CRDSA random access schemes, has been specifically studied to manage scenarios characterized by heterogeneity in the presence of time-disjoint transient links. The behavior of the system has been carefully evaluated by theoretically estimating the SINR distribution at each mmWave link to properly model the capture effect. A novel BA-CGR algorithm has been designed and developed to jointly consider the buffer occupancy constraints at the nSAT and the connection planning during the BS-nSAT contact periods. The designed architecture, which has been implemented in a developed discrete-time discrete-event simulator based on Matlab and NS3, has been applied to traffic scenarios with different requirements, discussing specific mechanisms to control the loss/delay tradeoff and exploring the impact of ISLs on the achievable performance.

The results have shown that an accurate model of the SINR distribution for mmWave terrestrial and satellite links may be obtained through formulations of limited mathematical complexity. Moving from these statistics, it has been shown that significant throughput improvements can be achieved by advanced Aloha-based schemes exploiting IC when the capture event is realistically taken into account. The simulation of the complete designed DTN architecture has proved the effectiveness of the BA-CGR algorithm, which completely avoids losses due to nSAT buffer overflow. Moreover, it has been shown that the resulting increase of the end-to-end delay with respect to CGR can be constrained by combining BA-CGR with proper dropping strategies, which can provide delay reductions comparable to those obtained enlarging the constellation size. The further delay decrease achieved in the presence ISLs finally confirms the advantages deriving from the integration between space and terrestrial mmWave systems, thus fostering the technological convergence between forthcoming $5 \mathrm{G}$ devices and expected K-band-based nSATs.

The proposed solution would represent a starting point for the near future employment of satellite networks in 5G environment. For this reason, current authors' efforts are devoted to a better integration with the envisioned $5 \mathrm{G}$ infrastructure and to the implementation of the SDN and NFV paradigms. Further ongoing investigation are focused on the additional aspects that could affect the obtained performance, such as the packet fragmentation and aggregation mechanisms, and the design of a complete ISL constellation configuration with both intra- and inter-orbit links.

\section{REFERENCES}

[1] M.N. Sweeting, "Modern small satellites-changing the economics of space," Proc. IEEE, vol. 106, no. 3, pp. 343-361, Mar. 2018.

[2] J.A. King, J. Ness, G. Bonin, M. Brett, and D. Faber, "Nanosat Ka-band communications - A paradigm shift in small satellite data throughput," in AIAA/USU Conf. Small Satellites, 2012.

[3] R.E. Hodges, D.J. Hoppe, M.J. Radway, and N.E. Chahat, "Novel deployable reflectarray antennas for CubeSat communications," in IEEE MTT-S Int. Microw. Symp., 2015.

[4] G. Buttazzoni, M. Comisso, A. Cuttin, M. Fragiacomo, R. Vescovo, and R. Vincenti Gatti, "Reconfigurable phased antenna array for extending cubesat operations to Ka-band: Design and feasibility," Acta Astronaut., vol. 137, pp. 114-121, 2017.

[5] B. Klofas and K. Leveque, "The future of CubeSat communications: Transitioning away from amateur radio frequencies for high-speed downlinks," in AMSAT Space Symp., 2012.

[6] A. Cuttin, F. Alimenti, F. Coromina, E. De Fazio, F. Dogo, M. Fragiacomo, P. Gervasoni, G. Gotti, A. Gregorio, P. Mezzanotte, E. Pagana, V. Palazzi, F. Pelusi, P. Petrini, L. Roselli, and R. Vincenti Gatti, "A Kaband transceiver for CubeSat satellites: Feasibility study and prototype development," in EuMC, 2018.

[7] F. Babich, M. Comisso, A. Crismani, and A. Dorni, "On the design of MAC protocols for multi-packet communication in IEEE 802.11 heterogeneous networks using adaptive antenna arrays," IEEE Trans. Mobile Comput., vol. 14, no. 11, pp. 2332-2348, Nov. 2015. 
[8] J.G. Andrews, S. Buzzi, W. Choi, S.V. Hanly, A. Lozano, A.C.K. Soong, and J.C. Zhang, "What will 5G be?" IEEE J. Sel. Areas Commun., vol. 32, no. 6, pp. 1065-1082, Jun. 2014.

[9] NetWorld2020s-SatCom WG, "The role of satellites in 5G," white paper, 2014.

[10] M. Jia, X. Gu, Q. Guo, W. Xiang, and N. Zhang, "Broadband hybrid satellite-terrestrial communication systems based on cognitive radio toward 5G," IEEE Wireless Commun., vol. 23, no. 6, pp. 96-106, Dec. 2016.

[11] X. Artiga, J. Núñez-Martínez, A. Pérez-Neira, G.J.L. Vela, and J.M.F. García, "Terrestrial-satellite integration in dynamic 5G backhaul networks," in IEEE ASMS/SPSC, 2016.

[12] J. Zhang, X. Zhang, M.A. Imran, B. Evans, Y. Zhang, and W. Wang, "Energy efficient hybrid satellite terrestrial 5G networks with software defined features," J. Commun. Netw., vol. 19, no. 2, pp. 147-161, Apr. 2017.

[13] S. Xu, X.-W. Wang, and M. Huang, "Software-defined next-generation satellite networks: Architecture, challenges, and solutions," IEEE Access, vol. 6, pp. 4027-4041, 2018

[14] S. Kim, E. Visotsky, P. Moorut, K. Bechta, A. Ghosh, and C. Dietrich, "Coexistence of $5 \mathrm{G}$ with the incumbents in the 28 and $70 \mathrm{GHz}$ bands," IEEE J. Sel. Areas Commun., vol. 35, no. 6, pp. 1254-1268, Jun. 2017.

[15] E. Casini, R. De Gaudenzi, and O. del Rio Herrero, "Contention resolution diversity slotted ALOHA (CRDSA): An enhanced random access scheme for satellite access packet networks," IEEE Trans. Wireless Commun., vol. 6, no. 4, pp. 1408-1419, Apr. 2007.

[16] Second gener. DVB interact. satellite syst. (DVB-RCS2): Lower layers for satellite std, ETSI EN 301 545-2 V1.2.1, 2014.

[17] E. Paolini, G. Liva, and M. Chiani, "Coded slotted ALOHA: A graphbased method for uncoordinated multiple access," IEEE Trans. Inf. Theory, vol. 61, no. 12, pp. 6815-6832, Dec. 2015.

[18] A. Osseiran et al., 5G mobile and wireless communications technology. Cambridge Univ. Press, Cambridge, UK, 2016.

[19] V. Cerf, S. Burleigh, A. Hooke, L. Torgerson, R. Durst, K. Scott, K. Fall, and H. Weiss, "Delay-tolerant networking architecture," RFC 4838, 2007.

[20] K. Fall, "A delay-tolerant network architecture for challenged internets," in ACM SIGCOMM, 2003.

[21] C. Caini, H. Cruickshank, S. Farrell, and M. Marchese, "Delay-and disruption-tolerant networking (DTN): An alternative solution for future satellite networking applications," Proc. IEEE, vol. 99, no. 11, pp. 1980 1997, Nov. 2011

[22] S. Jain, K. Fall, and R. Patra, "Routing in a delay tolerant network," in ACM SIGCOMM, 2004

[23] M. Marchese, F. Patrone, and M. Cello, "DTN-based nanosatellite architecture and hot spot selection algorithm for remote areas connection," IEEE Trans. Veh. Technol., vol. 67, no. 1, pp. 689-702, Jan. 2018.

[24] R. Radhakrishnan, W. Edmonson, F. Afghah, R.M. Rodriguez-Osorio, F. Pinto, and S.C. Burleigh, "Survey of inter-satellite communication for small satellite systems: Physical layer to network layer view," IEEE Commun. Surveys Tuts., vol. 18, no. 4, pp. 2442-2473, 2016.

[25] S. Burleigh, K. Fall, and E. Birrane, "Bundle protocol Version 7," Internet-Draft, draft-ietf-dtn-bpbis-11, 2018.

[26] S. Burleigh, "Contact graph routing," Internet-Draft, 2010

[27] Transport network support of IMT-2020/5G, ITU-T SG15, 2018.

[28] "Open source MANO," http://www.etsi.org/technologiesclusters/technologies/nfv/open-source-mano.

[29] L. Boero, R. Bruschi, F. Davoli, M. Marchese, and F. Patrone, "Satellite networking integration in the $5 \mathrm{G}$ ecosystem: Research trends and open challenges," IEEE Netw., vol. 32, no. 5, pp. 9-15, Sep. 2018

[30] International Telecommunication Union - Radiocommunication Study Groups (ITU-R), "Framework and overall objectives of the future development of IMT for 2020 and beyond," M.2083-0, 2015.

[31] S.-Y. Li and C.H. Liu, "An analytical model to predict the probability density function of elevation angles for LEO satellite systems," IEEE Commun. Lett., vol. 6, no. 4, pp. 138-140, Apr. 2002.

[32] G.D. Gordon and W.L. Morgan, Principles of communications satellites. New York, John Wiley and Sons, 1993.

[33] M.R. Akdeniz, Y. Liu, M.K. Samimi, S. Sun, S. Rangan, T.S. Rappaport, and E. Erkip, "Millimeter wave channel modeling and cellular capacity evaluation," IEEE J. Sel. Areas Commun., vol. 32, no. 6, pp. 1164-1179, Jun. 2014

[34] R. Abelló et al., "Low-Earth orbit (LEO) $26 \mathrm{GHz}$ K-band study group final report," Interagency Operat. Advisory Group, Tech. Rep., 2016.

[35] M. Di Renzo, "Stochastic geometry modeling and analysis of multitier millimeter wave cellular networks," IEEE Trans. Wireless Commun. vol. 14 , no. 9, pp. 5038-5057, Sep. 2015.
[36] T. Bai and R.W. Heath Jr., "Coverage and rate analysis for millimeterwave cellular networks," IEEE Trans. Wireless Commun., vol. 14, no. 2, pp. 1100-1114, Feb. 2015

[37] H. Shokri-Ghadikolaei and C. Fischione, "The transitional behavior of interference in millimeter wave networks and its impact on medium access control," IEEE Trans. Commun., vol. 64, no. 2, pp. 723-740, Feb. 2016.

[38] F. Babich, A. Soranzo, and F. Vatta, "Useful mathematical tools for capacity approaching codes design," IEEE Commun. Lett., vol. 21, no. 9 , pp. 1949-1952, Sep. 2017.

[39] Second gener. std. for broadband satellite serv. (DVB-S2), ETSI EN 302 307 V1.2.1, 2009.

[40] G. Araniti, N. Bezirgiannidis, E. Birrane, I. Bisio, S. Burleigh, C. Caini, M. Feldmann, M. Marchese, J. Segui, and K. Suzuki, "Contact graph routing in DTN space networks: Overview, enhancements and performance," IEEE Commun. Mag., vol. 53, no. 3, pp. 38-46, Mar. 2015.

[41] "Network Simulator 3," Accessed: 2018-11-28. [Online]. Available: \{https://www.nsnam.org/\}

[42] D. Vallado, P. Crawford, R. Hujsak, and T. Kelso, "Revisiting spacetrack report No. 3," in AIAA/AAS Astrodynam. Specialist Conf. Exhibit, 2006.

[43] Chen, Thomas M, "Network traffic modeling," The handbook of computer networks, vol. 3, 2007

[44] F. Babich and M. Comisso, "Including the angular domain in the analysis of finite multi-packet peer-to-peer networks with uniformly distributed sources," IEEE Trans. Commun., vol. 64, no. 6, pp. 2494-2510, Jun. 2016.

[45] R.D. Yates and D.J. Goodman, Probability and stochastic processes. New York, John Wiley and Sons, 1999.

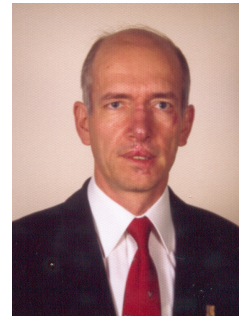

Fulvio Babich (SM'03) received the doctoral degree in electrical engineering, from the University of Trieste (Italy), in 1984. He worked for Telettra on optical communications and with Zeltron (Electrolux group) as Company Head in the Home System European projects. Currently, he is Associate Professor of digital communications at the Department of Engineering and Architecture of the University of Trieste. Fulvio Babich has served as co-chair for the Communication Theory Symposia at ICC 2005 and 2014, and for the Wireless Communication Symposia at ICC 2011 and WCSP 2012. Fulvio Babich has been member of the Directive Board of CNIT (National Inter-University Consortium for Telecommunications, a non-profit Consortium among 37 Universities, whose main purpose is to coordinate and foster basic and applied research).

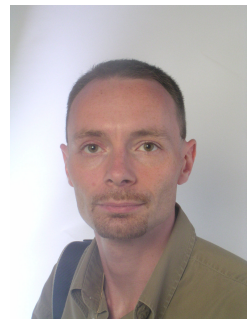

Massimiliano Comisso (M'09) received the Laurea and Ph.D. degrees in Information Engineering from the University of Trieste (Italy). He worked for Alcatel on DWDM systems and collaborated with Danieli Automation on NDE techniques. Currently, Massimiliano Comisso is Assistant Professor at the Department of Engineering and Architecture of the University of Trieste. He is author/co-author of more than 60 international scientific papers, and serves as referee/TPC member for several IEEE journals and conferences. He has been Best Student Paper Award Finalist at GLOBECOM 2006 and received the Best Paper Award at CAMAD 2009. His research interests involve smart antenna systems, distributed wireless networks, millimeter-wave communications, antenna array synthesis, and small antennas.

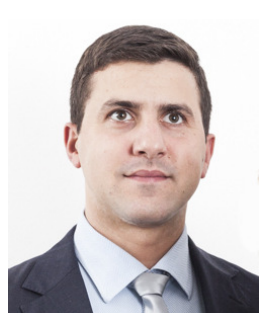

Alessandro Cuttin received the Ph.D. in Information Engineering and the M.Eng. in Telecommunication Engineering from the University of Trieste (Italy). He is co-founder of PicoSaTS s.r.l., a sinoff company of the University of Trieste. He has been with Esteco s.p.a. in 2014, fellow of the National Institute for Astrophysics (INAF) in 2008, and deputy project manager of the AtmoCube team, a CubeSat project of the University of Trieste. His research interests involve random access solutions and millimeter-wave antenna arrays for LEO satellites, and multi-objective optimization. 


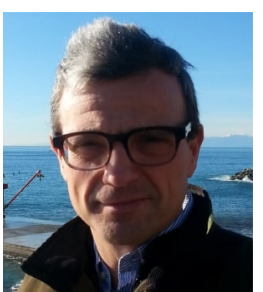

Mario Marchese (SM'04) was born in Genoa, Italy in 1967. He got his "Laurea" degree cum laude at the University of Genoa, Italy in 1992, and his Ph.D. (Italian "Dottorato di Ricerca") degree in "Telecommunications" at the University of Genoa in 1997. From 1999 to January 2005, he worked with the Italian Consortium of Telecommunications (CNIT), by the University of Genoa Research Unit, where he was Head of Research. From February 2005 to January 2016 he was Associate Professor at the University of Genoa. Since February 2016 he has been Full Professor at the University of Genoa. He authored/co-authored more than 300 scientific works, including international journals, international conference proceedings and book chapters. His main research activity concerns: networking, quality of service over heterogeneous networks, software defined networking, satellite DTN and nanosatellite networks, network security.

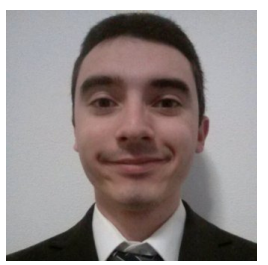

Fabio Patrone got his B.Sc. and his M.Sc. in Telecommunication Engineering in 2010 and 2013 at the University of Genoa. He got his Ph.D. at the Satellite Communications and Networking Laboratory (SCNL) with a thesis on routing and scheduling algorithms in satellite DTNs. He is currently a PostDoc research fellow at the SCNL. His main research activity concerns satellite networks, with particular regard to the design of routing, scheduling, and congestion control algorithms. 